\title{
Group valued null sequences and metrizable non-Mackey groups
}

\author{
Dikran Dikranjan, Elena Martín-Peinador and Vaja Tarieladze
}

Communicated by Karl-Hermann Neeb

\begin{abstract}
For a topological abelian group $X$ we topologize the group $c_{0}(X)$ of all $X$-valued null sequences in a way such that when $X=\mathbb{R}$ the topology of $c_{0}(\mathbb{R})$ coincides with the usual Banach space topology of the classical Banach space $c_{0}$. If $X$ is a nontrivial compact connected metrizable group, we prove that $c_{0}(X)$ is a non-compact Polish locally quasi-convex group with countable dual group $c_{0}(X)^{\wedge}$. Surprisingly, for a compact metrizable $X$, countability of $c_{0}(X)^{\wedge}$ leads to connectedness of $X$.

Our principal application of the above results is to the class of locally quasi-convex Mackey groups (LQC-Mackey groups). A topological group $(G, \mu)$ from a class $\mathcal{E}$ of topological abelian groups will be called a Mackey group in $\mathcal{E}$ or a $\mathscr{E}$-Mackey group if it has the following property: if $v$ is a group topology in $G$ such that $(G, v) \in \mathscr{Y}$ and $(G, v)$ has the same character group as $(G, \mu)$, then $v \leq \mu$.

Based upon the results obtained for $c_{0}(X)$, we provide a large family of metrizable precompact (hence, locally quasi-convex) connected groups which are not LQC-Mackey. Namely, we show that for a connected compact metrizable group $X \neq\{0\}$, the group $c_{0}(X)$, endowed with the topology induced from the product topology on $X^{\mathbb{N}}$, is a metrizable precompact connected group which is not a Mackey group in LQC.

Since metrizable locally convex spaces always carry the Mackey topology - a wellknown fact from Functional Analysis -, our results prove that a Mackey theory for abelian groups is not a simple traslation of items known to hold for locally convex spaces. This paper is a contribution to the Mackey theory for groups, where properties of a topological nature like compactness or connectedness have an important role.
\end{abstract}

Keywords. Mackey group, Mackey topology, precompact group, locally quasi-convex group, groups of null sequences, $c_{0}(X)$, summable sequence.

2010 Mathematics Subject Classification. Primary 54C40, 14E20; secondary 46E25, $20 \mathrm{C} 20$.

The authors acknowledge support of the MICINN of Spain through the grants MTM2009-14409C02-01 for the first author and MTM2009-14409-C02-02 (subprograma MTM) for the second and third ones; V. Tarieladze was also partially supported by Shota Rustaveli National Sciences Foundation grants GNSF/ST08/3-384 and GNSF/ST09/99-3-104. The three authors also acknowledge to the IMI (Institute for Interdisciplinary Mathematics) for supporting several visits of Dikranjan and Tarieladze to the Complutense University of Madrid, which have allowed to carry out this joint research. 


\section{Introduction}

All considered groups will be abelian. For groups $X, Y$ we denote by $\operatorname{Hom}(X, Y)$ the set of all group homomorphisms from $X$ to $Y$. Note that $\operatorname{Hom}(X, Y)$ is a group with respect to the pointwise addition/multiplication of homomorphisms. If $X, Y$ are topological groups, $\mathrm{CHom}(X, Y)$ stands for the subgroup of continuous elements of $\operatorname{Hom}(X, Y)$. A set $\Gamma \subset \operatorname{Hom}(X, Y)$ will be called separating if for every pair $x_{1}, x_{2}$ of distinct elements of $X$ there exists $\gamma \in \Gamma$ with $\gamma\left(x_{1}\right) \neq \gamma\left(x_{2}\right)$.

The set

$$
\mathbb{S}:=\{s \in \mathbb{C}:|s|=1\}
$$

is an abelian group with respect to multiplication of complex numbers; it is endowed with the usual topology induced from $\mathbb{C}$. For a group $G$ an element of $\operatorname{Hom}(G, \mathbb{S})$ is called a (multiplicative) character. If $G$ is a topological group, we write

$$
G^{\wedge}:=\operatorname{CHom}(G, \mathbb{S}),
$$

and we call an element of $G^{\wedge}$ a continuous character. Always $1 \in G^{\wedge}$, where $1(x)=1 \in \mathbb{S}, \forall x \in G$. This is the neutral element of $G^{\wedge}$. The group $G^{\wedge}$ (without any topology fixed in advance on it) is called the topological dual or the character group of $G$.

A topological group $G$ is called maximally almost periodic, for short a MAPgroup, if $G^{\wedge}$ is separating; $G$ is called minimally almost periodic if $G^{\wedge}=\{1\}$. We denote by MAP the class of all MAP-groups and by MinAP the class of all minimally almost periodic groups.

For a topological group $(G, \tau)$ the Bohr topology $\tau^{+}$is the initial topology for the set of all characters $\gamma: G \rightarrow \mathbb{S}, \gamma \in(G, \tau)^{\wedge}$. Clearly $\tau^{+} \leq \tau$ and $\tau^{+}$is a Hausdorff topology iff $(G, \tau)$ is MAP. In such a case, $(G, \tau)$ is precompact iff $\tau=\tau^{+}$(see also Example 1.2).

\section{Connectedness and smallness of the dual of the group of null sequences}

Convergent sequences are of primary importance in topological groups. For a topological group $X$, denote by $c_{0}(X)$ the subgroup of $X^{\mathbb{N}}$ of all null sequences of $X$ and let $\mathfrak{u}_{0}$ be the uniform topology induced from $X^{\mathbb{N}}$ on $c_{0}(X)$ (see Section 3 for the relevant definitions). Clearly, $c_{0}(X)$ coincides with the direct $\operatorname{sum} X^{(\mathbb{N})}$ precisely when $X$ has no non-trivial convergent sequences. The passage from $X$ to $\left(c_{0}(X), \mathfrak{u}_{0}\right)$ preserves (sequential) completeness, metrizability, separability, MAP, local quasi-convexity (see Definition 2.1), non-discreteness, and connectedness (see Proposition 3.4). 
Surprisingly, for a compact metrizable abelian group $X$ and

$$
G=\left(c_{0}(X), \mathfrak{u}_{0}\right),
$$

countability of $G^{\wedge}$ (or even the milder condition $\left|G^{\wedge}\right|<c$ ) leads to connectedness of $X$ :

Main Theorem. Assume that $X$ is an infinite compact metrizable topological abelian group. Then $G:=\left(c_{0}(X), \mathfrak{u}_{0}\right)$ is a nonprecompact locally quasi-convex Polish group. Further, the following assertions are equivalent:

(i) $X$ is connected.

(ii) $G^{\wedge}$ is isomorphic to a direct sum of countable many copies of $X^{\wedge}$.

(iii) $G^{\wedge}$ is countable.

(iv) $\left|G^{\wedge}\right|<\mathrm{c}$.

If the above conditions hold true, then $G$ itself is connected.

We show in Remark 3.5 that the assignment

$$
X \mapsto \mathcal{F} X:=\left(c_{0}(X), \mathfrak{u}_{0}\right)
$$

defines a functor $\mathscr{F}: \mathfrak{H} \rightarrow \mathfrak{H}$, where $\mathfrak{H}$ is the category of Hausdorff topological abelian groups and continuous homomorphism. This functor has the following property: if $X$ is a non-trivial connected compact metrizable abelian group, then $\mathscr{F} X$ is a connected, non-precompact locally quasi-convex Polish group with countable dual.

To the proof of the Main Theorem (together with other more precise results) are dedicated Sections 3-6. The backbone of the proof is the class $\mathfrak{B}$ of topological groups $X$ such that for every non-trivial $\xi \in X^{\wedge}$ there exists a null sequence $\left(x_{n}\right)$ in $X$ such that the sequence $\left(\prod_{k=1}^{n} \xi\left(x_{k}\right)\right)$ is not convergent in $\mathbb{S}$ (see Definition 6.1). Clearly, $\mathfrak{B}$ contains all minimally almost periodic groups. A precompact group $X$ belongs to $\mathfrak{B}$ precisely when $c_{0}(X)^{\wedge}=\left(X^{\wedge}\right)^{(\mathbb{N})}$ (Theorem 6.3). It turns out that this new class $\mathfrak{B}$ can be described in some cases through well-known topological properties close to connectedness. For instance, in Corollary 6.13 we show that in the class LCA of all locally compact groups, $X \in \mathfrak{B}$ iff $X$ is connected.

Furthermore, a weaker form of connectedness characterizes $\mathfrak{B}$ within the class of metrizable groups. Namely, Enflo [17] introduced the notion of locally generated groups (see Definition 6.8; according to Lemma 6.9, these are precisely the groups without proper open subgroups, in particular connected groups are locally generated). We prove in Theorem 6.12 that every group in $\mathfrak{B}$ is locally generated, while all metrizable locally generated groups belong to the class $\mathfrak{B}$. In the next di- 
agram we collect the implications (denoted by solid arrows) between all the above mentioned properties with particular emphasis on the class $\mathfrak{B}$ (placed in the center):

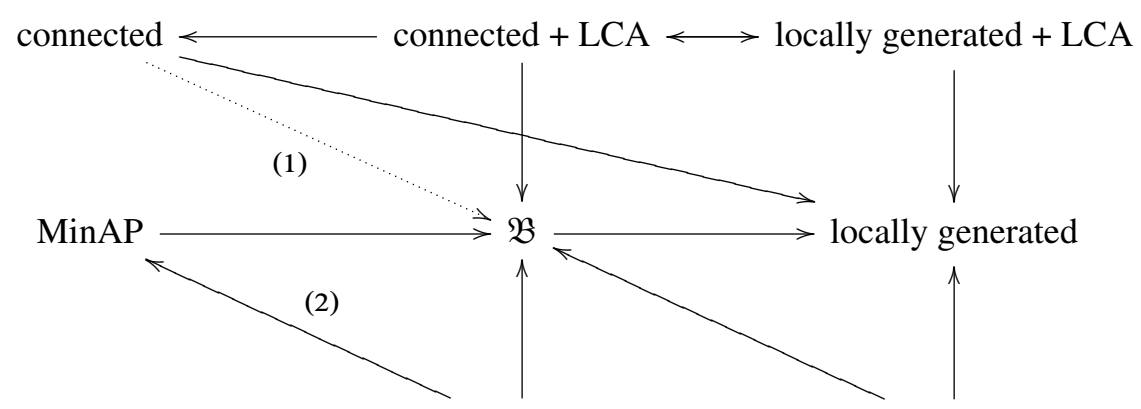

$\mathfrak{B} \&$ no conv. sequences locally generated + metrizable

The easy implication (2) is proved in Proposition 6.5. It implies that non-trivial precompact groups without non-trivial convergent sequences do not belong to $\mathfrak{B}$. Remark 6.16 provides an example of a connected precompact group not in $\mathfrak{B}$, witnessing thereby the missing implication (1). Obviously, the failure of this implication shows that locally generated $\nRightarrow \mathfrak{B}$.

The class $\mathfrak{B}$ has nice stability properties: it is stable under taking continuous homomorphic images and arbitrary direct products, if $H$ is a dense subgroup of a group $G$, then $H \in \mathfrak{B}$ yields $G \in \mathfrak{B}$. Dense subgroups of metrizable $\mathfrak{B}$-groups have the property $\mathfrak{B}$ (by the characterization of the metrizable $\mathfrak{B}$-groups in Theorem 6.12), yet there exist dense connected subgroups $H \notin \mathfrak{B}$ of a compact connected group $G$ (so $G \in \mathfrak{B}$ ), according to Remark 6.16.

\section{Mackey groups and metrization}

The principal application of the above results is in the field of Mackey groups. The aim of the present subsection is to introduce this fascinating topic and the specific problem we resolve by means of our Main Theorem.

The notion of a Mackey group is inspired by the concept of a Mackey space in the theory of topological vector spaces. Recall that for a topological vector space $E$ over $\mathbb{R}$ the dual space $E^{*}$ consists of all continuous linear functionals $f: E \rightarrow \mathbb{R}$. The Mackey-Arens Theorem establishes that for a topological vector space $E$ over $\mathbb{R}$ there always exists a finest topology in the class of locally convex vector space topologies giving rise to the same dual space $E^{*}$. This topology is named as the Mackey topology of $E$. A topological vector space $E$ over $\mathbb{R}$ is a Mackey space iff its original topology coincides with the Mackey topology of $E$. Note that the 
concept of a Mackey space can be defined directly, i.e. without a reference to the Mackey-Arens Theorem as follows: A topological vector space $E$ over $\mathbb{R}$ is a Mackey space iff it is locally convex and its original topology is the finest one in the class of locally convex vector topologies giving rise to the same dual space $E^{*}$.

The first attempts to obtain results of Mackey-Arens type for topological abelian groups were done in [28] for the class of locally precompact group topologies, and in [10] for the class of locally quasi-convex group topologies. As pointed out in [10], the Mackey-Arens Theorem gives rise to two non-equivalent definitions of the Mackey topology for a locally quasi-convex group. Although they are compared and several important results are established, it is not explicitly fixed in [10] which of them should be called the Mackey topology for an LQC-group. This is done in the present paper, from a more categorical point of view, which specialized for LQC-groups could be the "missing definition" of [10].

Definition 1.1. If $(G, \tau)$ is a topological group, a group topology $\eta$ in $G$ is said to be compatible for $(G, \tau)$ if $(G, \tau)^{\wedge}=(G, \eta)^{\wedge}$.

Example 1.2 ([11]; also [15, Theorem 2.3.4] and [28]). For a MAP-group $(G, \tau)$, $\tau^{+}$is a precompact compatible group topology, i.e., $(G, \tau)^{\wedge}=\left(G, \tau^{+}\right)^{\wedge}$.

The following definition is in the spirit of [5], where a categorical treatment of Mackey groups is given.

Definition 1.3. Let $\mathcal{G}$ be a class of topological abelian groups.

(a) A topological group $(G, \mu)$ is called a Mackey group in $\mathcal{E}$ if $(G, \mu) \in \mathcal{E}$ and if $v$ is a compatible group topology for $(G, \mu)$, with $(G, v) \in \mathscr{E}$, then $v \leq \mu$.

(b) Let $(G, v) \in \mathcal{E}$. If there exists a group topology $\mu$ in $G$ compatible for $(G, v)$ such that $(G, \mu)$ is a Mackey group in $\mathcal{G}$, then $\mu$ is called the $\mathcal{E}$-Mackey topology of $(G, v)$.

Let LPC denote the class of Hausdorff locally precompact topological abelian groups. If $(G, \tau) \in$ LPC is not precompact (e.g., if $G$ is discrete), then $\left(G, \tau^{+}\right) \in$ LPC is not a Mackey group in LPC. Hence, a precompact group in LPC need not be a Mackey group in LPC in general. However, the metrizability changes the picture as the following statement shows.

Theorem 1.4 (cf. [28, Corollary 2, p. 484]). Every metrizable $(G, \mu) \in$ LPC is a Mackey group in LPC.

Next we consider another class of groups in which the metrizable groups are again Mackey. Let LCS be the class of Hausdorff topological abelian groups which 
admit a structure of locally convex topological vector space over $\mathbb{R}$, loosely speaking LCS is the class of Hausdorff locally convex spaces considered just as additive groups. It is an important consequence of the Hahn-Banach Theorem that

\section{LCS $\subset$ MAP.}

Taking into account that the group of continuous linear forms on a group $G \in$ LCS is algebraically isomorphic to the group of its continuous characters, according to [25, IV 3.4], it is easy to derive the following:

Theorem 1.5. Every metrizable $(G, \mu) \in \mathrm{LCS}$ is a Mackey group in LCS.

A natural class of groups containing LPC $\cup$ LCS is provided by LQC, the class of locally quasi-convex Hausdorff groups (see Definition 2.1). In fact, it is known that

$$
\mathrm{LPC} \cup \mathrm{LCS} \subset \mathrm{LQC} \subset \mathrm{MAP}
$$

where the inclusions are strict (cf. [2,4,10]). It was proved in [10] that every $\check{C} e c h$ complete $(G, \mu) \in \mathrm{LQC}$ is a Mackey group in LQC. In particular, every locally compact $(G, \mu) \in \mathrm{LQC}$ is a Mackey group in LQC. We isolate here another particular case more relevant for the specific purposes of this paper:

Theorem 1.6 ([10]). Every complete metrizable $(G, \mu) \in \mathrm{LQC}$ is a Mackey group in LQC.

Let us discuss to what extent completeness is an essential assumption in Theorem 1.6.

Example 1.7. Let $(G, \tau)$ be a precompact group of finite exponent with $\left|G^{\wedge}\right|<c$. It is proved in [7] that $\tau$ is the only locally quasi-convex group topology compatible for $(G, \tau)$. Consequently, $(G, \tau)$ is LQC-Mackey. If $G$ is furthermore required to be countable and metrizable (e.g., the direct sum $\mathbb{Z}_{2}^{(\mathbb{N})}$ equipped with the product topology should do), then $G$ cannot be complete as non-discrete complete metrizable groups are second category, hence uncountable. This provides examples of non-complete countable precompact metrizable Mackey groups. Since every precompact group of finite exponent has a linear topology, all these groups are zero-dimensional, hence totally disconnected.

A large scale of examples of non-complete metrizable LQC-groups which are not Mackey was provided for the first time in a preliminary on-line version of the present paper, [14]. Soon after it was shown in [3] that $(\mathbb{Z}, \tau)$, where $\tau$ is a nondiscrete linear topology, is a non-complete countable precompact metrizable group which is not LQC-Mackey. 
The examples of metrizable non-Mackey groups provided by us in the present paper are based upon the following result (see the end of Section 2 for its proof):

Theorem 1.8. Let $(G, \tau) \in \mathrm{LQC}$ be a non-precompact group with countable dual $(G, \tau)^{\wedge}$. Then $\left(G, \tau^{+}\right)$is a metrizable precompact group which is not a Mackey group in LQC.

Examples of groups $(G, \tau)$ satisfying the assumptions of the previous theorem are not immediately at hand, since by Proposition 2.3 they cannot be locally precompact (even if they are LQC). Moreover, they cannot be LCS either, since only a trivial LCS can have a countable dual. The first example of such a group, namely $\left(c_{0}(\mathbb{S}), \mathfrak{u}_{0}\right)$, is given in Proposition 5.2 below and the existence of a large family of groups with this property is obtained by the general construction developed in our Main Theorem. Composing the functor $\mathcal{F}$ from equation (1.1) with the functor $(G, \tau) \mapsto\left(G, \tau^{+}\right)$of the Bohr modification, one obtains a functor $\mathcal{E}$ from the category $\mathfrak{C}$ of compact connected metrizable groups to the category $\mathfrak{P}_{\text {met }}$ of metrizable precompact abelian groups such that each $\mathcal{E}(X)$ is a connected precompact metrizable non-Mackey group whenever $X$ is non-trivial. By Remark 3.5 (c), for every non-trivial Polish LQC group $X$, the group $G=\mathcal{F} X$ is Polish, LQC and nonprecompact. By our Main Theorem, $G^{\wedge}$ is countable whenever $X$ is also compact and connected. Let us mention also, that the connectedness of $X$ yields as a byproduct also connectedness of both $G$ and $\mathscr{E}(X)$.

In the last section we offer some open questions and conjectures.

\section{Notation and terminology}

The symbols $\mathbb{N}, \mathbb{Z}, \mathbb{Q}, \mathbb{R}$ and $\mathbb{Z}_{m}$ are used for the set of naturals, the group of integers, the group of rationals, the group of reals and the cyclic group of size $m$, respectively. Let $\mathbb{S}$ denote the circle group and

$$
\mathbb{S}_{+}=\{s \in \mathbb{S}: \operatorname{Re}(s) \geq 0\} .
$$

The cardinality of the continuum will be denoted by $c$ and $|X|$ will stand for the cardinality of a set $X$.

For a topological space $X$ we denote by $c(X)$ the cellularity of $X$, this is the smallest cardinal $\kappa$ such that every family of non-empty pairwise disjoint open sets has size $\leq \kappa$.

We denote by $e$ the neutral element of a group. We also use the symbols 0 and 1 instead of $e$ if the group is known to be additive or multiplicative respectively. For a subset $A$ of a group $X$ denote by $\langle A\rangle$ the subgroup of $X$ generated by $A$.

Let $X$ be a set. As usual, $X^{\mathbb{N}}$ will denote the set of all sequences $\mathbf{x}=\left(x_{n}\right)_{n \in \mathbb{N}}$ of elements of $X$ and $\left(p_{n}\right)_{n \in \mathbb{N}}$ the sequence of projections $X^{\mathbb{N}} \rightarrow X$. 
For a group $X, X^{(\mathbb{N})}$ will be the subgroup of $X^{\mathbb{N}}$ consisting of all sequences eventually equal to $e$. For $n \in \mathbb{N}$ define an injective homomorphism

$$
v_{n}: X \rightarrow X^{(\mathbb{N})} \text { by } v_{n}(x)=(e, \ldots, e, x, e, \ldots),
$$

where $x \in X$ is placed in position $n$.

If $X$ is a topological group, let

$$
c_{0}(X):=\left\{\left(x_{n}\right)_{n \in \mathbb{N}} \in X^{\mathbb{N}}: \lim _{n} x_{n}=e\right\} .
$$

Clearly $c_{0}(X)$ is a subgroup of $X^{\mathbb{N}}$ containing $X^{(\mathbb{N})} ;$ moreover, $c_{0}(X)=X^{(\mathbb{N})}$ iff $X$ has only trivial convergent sequences.

For a topological group $X, \mathcal{N}(X)$ is the set of all neighborhoods of $e \in X$. If $\mathcal{N}(X)$ admits a base consisting of open subgroups, we say that $X$ is linear (and its topology is linear). Clearly, $\mathbb{S}_{+} \in \mathcal{N}(\mathbb{S})$. We write $X_{\text {co }}^{\wedge}$ for the group $X^{\wedge}$ endowed with the compact-open topology. For a subset $V$ of $X$ let

$$
V^{\triangleright}:=\left\{\xi \in X^{\wedge}: \xi(V) \subset \mathbb{S}_{+}\right\} .
$$

If $X, Y$ are topological abelian groups and $\varphi \in \operatorname{CHom}(X, Y)$, then the mapping $\varphi^{\wedge}: Y^{\wedge} \rightarrow X^{\wedge}$, defined by $\varphi^{\wedge}(\eta)=\eta \circ \varphi$ for $\eta \in Y^{\wedge}$, is a group homomorphism called the dual homomorphism.

The von Neumann's kernel of a topological abelian group $X$ is defined by

$$
\mathbf{n}(X)=\bigcap\left\{\operatorname{ker} \xi: \xi \in X^{\wedge}\right\} .
$$

Clearly, $\mathbf{n}(X)$ is a subgroup of $X$, and $X$ is MAP iff $\mathbf{n}(X)=\{0\}$, while $X$ is $\operatorname{MinAP}$ iff $\mathbf{n}(X)=X$.

\section{Locally quasi-convex groups}

Let us recall the definition of a locally quasi-convex group.

Definition 2.1 ([29]). A subset $A$ of a topological group $G$ is called quasi-convex if for every $x \in G \backslash A$ there exists $\chi \in G^{\wedge}$ such that

$$
\chi(A) \subset \mathbb{S}_{+} \text {but } \chi(x) \notin \mathbb{S}_{+} .
$$

A topological group $G$ is called locally quasi-convex if $\mathcal{N}(G)$ admits a basis consisting of quasi-convex subsets of $G$.

The precompact and the locally precompact groups are prominent classes of locally quasi-convex groups. We characterize below the groups $X \in \mathrm{LPC}$ with countable duals. 
Proposition 2.2. Assume that $X$ is a precompact Hausdorff topological group and $V \in \mathcal{N}(X)$. Then $V^{\triangleright}$ is a finite subset of $X^{\wedge}$.

Proof. It is known that $V^{\triangleright}$ is a compact subset of $X_{\text {co }}^{\wedge}$. If $X$ is compact, then $X_{\text {co }}^{\wedge}$ is discrete and $V^{\triangleright}$ is finite in this case. If $X$ is not compact, then it is a dense subgroup of a compact Hausdorff topological group $K$, namely its completion. Let $U$ denote the closure of $V$ in $K$; clearly $U \in \mathcal{N}(K)$. Since $\mathbb{S}_{+}$is closed in $\mathbb{S}$, the density of $V$ in $U$ implies that

$$
V^{\triangleright}=\left\{\left.\xi\right|_{X}: \xi \in U^{\triangleright}\right\} .
$$

Now, $U^{\triangleright}$ finite implies that $V^{\triangleright}$ is finite as well.

Proposition 2.3. For an infinite locally precompact Hausdorff topological abelian group $X$ TFAE:

(i) $X$ is precompact metrizable.

(ii) $X^{\wedge}$ is countable.

Proof. (i) $\Longrightarrow$ (ii). This implication follows easily from Proposition 2.2 (also from $[20,(24.14)])$.

(ii) $\Longrightarrow$ (i). Let $Y$ be the completion of $X$. It is known that the groups $X^{\wedge}$ and $Y^{\wedge}$ are algebraically isomorphic, hence, $Y^{\wedge}$ is countable. On the other hand, $Y$ is a locally compact Hausdorff topological abelian group, therefore $Y_{\mathrm{co}}^{\wedge}$ is LCA. Since a second category countable Hausdorff topological group is discrete, we have that $Y_{\text {co }}^{\wedge}$ is a discrete countable group. Hence $\left(Y_{\mathrm{co}}^{\wedge}\right)_{\mathrm{co}}^{\wedge}$ is a compact metrizable group. By Pontryagin's theorem, $Y$ and $\left(Y_{\mathrm{co}}^{\wedge}\right)_{\mathrm{co}}^{\wedge}$ are topologically isomorphic. Thus $Y$ is compact metrizable and its topological subgroup $X$ is precompact metrizable.

Remark 2.4. (a) From Proposition 2.3 it follows that a compact non-metrizable group $X$ has a large dual, $\left|X^{\wedge}\right|>\boldsymbol{\aleph}_{0}$.

(b) The implication (i) $\Longrightarrow$ (ii) of Proposition 2.3 holds without any additional assumption on $X$. However (ii) $\Longrightarrow$ (i) may fail if $X$ is a locally quasi-convex Hausdorff group, as the Main Theorem shows.

Let us conclude this section with the proof of Theorem 1.8.

Proof of Theorem 1.8. Since $(G, \tau)^{\wedge}$ is countable, it follows that $\tau^{+}$is metrizable. The topology $\tau^{+}$is precompact and compatible for $(G, \tau)$ by Proposition 1.2. The group $\left(G, \tau^{+}\right) \in \mathrm{LQC}$ because precompact groups are locally quasi-convex. Since $(G, \tau)$ is not precompact, we have that $\tau^{+}<\tau$ being $\tau^{+} \neq \tau$. Hence $\left(G, \tau^{+}\right)$is a metrizable precompact group which is not a Mackey group in LQC. 


\section{Groups of sequences}

\section{The uniform topology in $X^{\mathbb{N}}$}

In what follows $X$ will be a fixed Hausdorff topological abelian group. We denote by $\mathfrak{p}_{X}$ the product topology in $X^{\mathbb{N}}$ and by $\mathfrak{b}_{X}$ the box topology in $X^{\mathbb{N}}$. It is easily verified that the collection

$$
\left\{V^{\mathbb{N}}: V \in \mathcal{N}(X)\right\}
$$

is a basis at $e$ for a group topology in $X^{\mathbb{N}}$ which we denote by $\mathfrak{u}_{X}$. In all three cases we shall omit the subscript $X$ when no confusion is likely.

The topology $u$ in $X^{\mathbb{N}}$ is nothing else but the topology of uniform convergence on $\mathbb{N}$ when the elements of $X^{\mathbb{N}}$ are viewed as functions from $\mathbb{N}$ to $X$ and $X$ is considered as a uniform space with respect to its left (= right) uniformity. So it will be called the uniform topology. Since it plays an important role in the sequel, we give in the next proposition an account of its main properties.

We write

$$
\mathfrak{p}_{0}:=\left.\mathfrak{p}\right|_{c_{0}(X)}, \quad \mathfrak{b}_{0}:=\left.\mathfrak{b}\right|_{c_{0}(X)} \quad \text { and } \quad \mathfrak{u}_{0}:=\left.\mathfrak{u}\right|_{c_{0}(X)} .
$$

Proposition 3.1. Let $(X,+)$ be a Hausdorff topological abelian group.

(a) The uniform topology $\mathfrak{u}$ is a Hausdorff group topology in $X^{\mathbb{N}}$ with $\mathfrak{p} \leq \mathfrak{u} \leq \mathfrak{b}$. Moreover,

$\left.\left(\mathrm{a}_{1}\right) \mathfrak{p}\right|_{X^{(\mathbb{N})}}=\left.\mathfrak{u}\right|_{X^{(\mathbb{N})}} \Longleftrightarrow X=\{0\}$.

$\left.\left(\mathrm{a}_{2}\right) \mathfrak{u}\right|_{X^{(\mathbb{N})}}=\left.\mathfrak{b}\right|_{X^{(\mathbb{N})}} \Longrightarrow X$ is a P-group $\Longrightarrow \mathfrak{u}=\mathfrak{b}$; in particular, if $X$ is metrizable and $\left.\mathfrak{u}\right|_{X^{(\mathbb{N})}}=\left.\mathfrak{b}\right|_{X^{(\mathbb{N})} \text {, then } X \text { is discrete. }}$

(b) The passage from $X$ to $\left(X^{\mathbb{N}}, \mathfrak{u}\right)$ preserves (sequential) completeness, metrizability, MAP and local quasi-convexity.

(c) If $X \neq\{0\}$, then:

$\left(\mathrm{c}_{1}\right) c\left(X^{\mathbb{N}}, \mathfrak{u}\right) \geq \mathfrak{c}$, in particular $\left(X^{\mathbb{N}}, \mathfrak{u}\right)$ is not separable.

$\left(c_{2}\right)\left(X^{(\mathbb{N})},\left.\mathfrak{u}\right|_{\left.X^{(\mathbb{N})}\right)}\right)$ is not precompact and hence $\left(c_{0}(X), \mathfrak{u}_{0}\right)$ and $\left(X^{\mathbb{N}}, \mathfrak{u}\right)$ are not precompact.

Proof. (a) The first assertion has a straightforward proof.

$\left(\mathrm{a}_{1}\right)$ Suppose that $X \neq\{0\}$. Take $x \in X \backslash\{0\}$. Then we have $v_{k}(x) \in X^{(\mathbb{N})}$, $k=1,2, \ldots$, and the sequence $\left(v_{k}(x)\right)_{k \in \mathbb{N}}$ tends to 0 in $\mathfrak{p}$. Since $X$ is Hausdorff, there is a $V \in \mathcal{N}(X)$ such that $x \notin V$. Then $v_{k}(x) \notin V^{\mathbb{N}}, k=1,2, \ldots$ Hence, the sequence $\left(v_{k}(x)\right)_{k \in \mathbb{N}}$ does not tend to 0 in $\mathfrak{u}$.

$\left(a_{2}\right)$ The second implication is straightforward. In order to verify the first one, suppose that $\left.\mathfrak{u}\right|_{X^{(\mathbb{N})}} \geq\left.\mathfrak{b}\right|_{X^{(\mathbb{N})}}$. Take arbitrarily $U_{n} \in \mathcal{N}(X), n=1,2, \ldots$ Then 
$\left(\prod_{n \in \mathbb{N}} U_{n}\right) \cap X^{(\mathbb{N})}$ is a neighborhood of zero in $\left.\mathfrak{b}\right|_{X^{(\mathbb{N})}}$. As $\left.\mathfrak{u}\right|_{X^{(\mathbb{N})}} \geq\left.\mathfrak{b}\right|_{X^{(\mathbb{N})}}$, there is a $V \in \mathcal{N}(X)$ such that

$$
V^{\mathbb{N}} \cap X^{(\mathbb{N})} \subset\left(\prod_{n \in \mathbb{N}} U_{n}\right) \cap X^{(\mathbb{N})} .
$$

From $v_{k}(V) \subseteq V^{\mathbb{N}} \cap X^{(\mathbb{N})}, k=1,2, \ldots$, we get $v_{k}(V) \subseteq\left(\prod_{n \in \mathbb{N}} U_{n}\right) \cap X^{(\mathbb{N})}$, $k=1,2, \ldots$ So, $V \subseteq U_{n}, n=1,2, \ldots$, therefore $V \subset \bigcap_{n \in \mathbb{N}} U_{n}$. Thus, for each sequence $U_{n} \in \mathcal{N}(X), n=1,2, \ldots, \bigcap_{n \in \mathbb{N}} U_{n} \in \mathcal{N}(X)$. Consequently, $X$ is a P-group.

The last assertion follows from the well-known fact that a metrizable P-space is discrete.

(b) We omit the standard proofs of the first two cases.

Assume that $X$ is MAP and let $\mathbf{x}=\left(x_{n}\right)_{n \in \mathbb{N}} \in X^{\mathbb{N}} \backslash\{\mathbf{0}\}$. Take $n \in \mathbb{N}$ such that $p_{n}(\mathbf{x}) \neq 0$. Since $X$ is MAP, there is $\xi \in X^{\wedge}$ such that $\xi\left(p_{n}(\mathbf{x})\right) \neq 1$. Clearly $p_{n}$ is $u$-continuous, therefore we get $\varphi:=\xi \circ p_{n} \in\left(X^{\mathbb{N}}, u\right)^{\wedge}$ and $\varphi(\mathbf{x}) \neq 1$. Hence, $\left(X^{\mathbb{N}}, \mathfrak{u}\right)$ is MAP.

In order to prove that $\left(X^{\mathbb{N}}, \mathfrak{u}\right)$ is locally quasi-convex provided that $X$ has the same property, just observe that for any quasi-convex $V \in \mathcal{N}(X)$,

$$
V^{\mathbb{N}}=\bigcap_{n \in \mathbb{N}} p_{n}^{-1}(V)
$$

and quasi-convexity is preserved under inverse images by continuous homomorphisms and under arbitrary intersections.

(c $1_{1}$ Let us fix $x \in X \backslash\{0\}$ and $U \in \mathcal{N}(X)$ such that $U \cap\{-x, x\}=\emptyset$. Write $C=\{0, x\}^{\mathbb{N}}$. Then

$$
\operatorname{card}(C)=c, \quad \mathbf{y}_{1} \in C, \mathbf{y}_{2} \in C, \mathbf{y}_{1} \neq \mathbf{y}_{\mathbf{2}} \Longrightarrow \mathbf{y}_{1}-\mathbf{y}_{2} \notin U^{\mathbb{N}} .
$$

Take a symmetric open $V \in \mathcal{N}(X)$ such that $V+V \subset U$. For every $\mathbf{y} \in C$ the non-empty set $V_{\mathbf{y}}=\mathbf{y}+V$ is open and for $\mathbf{y}_{\mathbf{1}} \in C, \mathbf{y}_{\mathbf{2}} \in C, \mathbf{y}_{\mathbf{1}} \neq \mathbf{y}_{\mathbf{2}}$ one can deduce $V_{\mathbf{y}_{1}} \cap V_{\mathbf{y}_{2}}=\varnothing$ from (3.1) and $V+V \subset U$. Hence the family $\left\{V_{\mathbf{y}}: \mathbf{y} \in C\right\}$ witnesses $c\left(X^{\mathbb{N}}, \mathfrak{u}\right) \geq c$.

$\left(\mathrm{c}_{2}\right)$ Fix $x \in X, x \neq 0$ and a symmetric $V \in \mathcal{N}(X)$ such that $x \notin V$. Then

$$
m, n \in \mathbb{N}, m \neq n \Longrightarrow v_{m}(x)-v_{n}(x) \notin V^{\mathbb{N}} \text {. }
$$

Now (3.2) together with the fact that $v_{m}(x)-v_{n}(x) \in X^{(\mathbb{N})}, \forall m, n \in \mathbb{N}$, yield that $\left(X^{(\mathbb{N})},\left.\mathfrak{u}\right|_{X^{(\mathbb{N})}}\right)$ is not precompact.

Remark 3.2. If $X$ is a compact metrizable group and $\rho$ is an invariant metric for $X$, then the equality

$$
d_{\infty}(\mathbf{x}, \mathbf{y})=\sup _{n \in \mathbb{N}} \rho\left(x_{n}, y_{n}\right), \quad \mathbf{x}, \mathbf{y} \in X^{\mathbb{N}},
$$


defines an invariant metric for $\left(X^{\mathbb{N}}, \mathfrak{u}\right)$. In particular, the topology of $\left(\mathbb{S}^{\mathbb{N}}, \mathfrak{u}\right)$ can be induced by the following metric:

$$
d_{\infty}(\mathbf{x}, \mathbf{y})=\sup _{n \in \mathbb{N}}\left|x_{n}-y_{n}\right|, \quad \mathbf{x}, \mathbf{y} \in \mathbb{S}^{\mathbb{N}} .
$$

According to [12, Example 4.2] the metric group $\left(\mathbb{S}^{\mathbb{N}}, d_{\infty}\right)$ has the following remarkable property: it is not precompact, but every uniformly continuous realvalued function defined on it is bounded.

\section{The group of null sequences $c_{0}(X)$}

In this section we study the group $c_{0}(X)$ of all null sequences of a topological abelian group $X$ as a subgroup of $\left(X^{\mathbb{N}}, \mathfrak{u}\right)$. It is easy to prove the following:

Lemma 3.3. Let $X$ be a topological group. The following assertions hold:

(a) $c_{0}(X)$ is closed in $\left(X^{\mathbb{N}}, \mathfrak{u}\right)$.

(b) If $\mathbf{x}=\left(x_{n}\right)_{n \in \mathbb{N}} \in c_{0}(X)$, then the sequence $\left(\sum_{k=1}^{n} v_{k}\left(x_{k}\right)\right)_{n \in \mathbb{N}}$ converges to $\mathbf{x}$ in the topology $\mathfrak{u}$; in particular, $X^{(\mathbb{N})}$ is a $\mathfrak{u}$-dense subset of $c_{0}(X)$.

Thus, summarizing:

$$
X^{(\mathbb{N})} \stackrel{\mathfrak{u} \text {-dense }}{\subset} c_{0}(X) \stackrel{\mathfrak{u} \text {-closed }}{\subset} X^{\mathbb{N}} .
$$

Since the groups of the form $\left(c_{0}(X), \mathfrak{u}_{0}\right)$ are the main object of our future considerations, we describe explicitly some of their properties, inherited from $\left(X^{\mathbb{N}}, \mathfrak{u}\right)$, or lifted from properties of $X^{(\mathbb{N})}$.

Proposition 3.4. Let $X$ be a Hausdorff topological abelian group.

(a) $\left(c_{0}(X), \mathfrak{u}_{0}\right)$ is a Hausdorff topological group having as a basis at zero the collection $\left\{V^{\mathbb{N}} \cap c_{0}(X): V \in \mathcal{N}(X)\right\}$.

(b) $\mathfrak{p}_{0} \leq \mathfrak{u}_{0} \leq \mathfrak{b}_{0}$. Moreover, we have $\mathfrak{p}_{0}=\mathfrak{u}_{0} \Longleftrightarrow X=\{0\}$; if $X$ is metrizable and $\mathfrak{u}_{0}=\mathfrak{b}_{0}$, then $X$ is discrete.

(c) The passage from $X$ to $\left(c_{0}(X), \mathfrak{u}_{0}\right)$ preserves (sequential) completeness, metrizability, separability, MAP, local quasi-convexity, non-discreteness, and connectedness.

Proof. (c) Assume $X$ is separable. The density of $X^{(\mathbb{N})}$ in $c_{0}(X)$ yields that $c_{0}(X)$ is also separable. If $\left(c_{0}(X), \mathfrak{u}_{0}\right)$ is discrete, for some $V \in \mathcal{N}(X)$, we have that

$$
V^{\mathbb{N}} \cap c_{0}(X)=\{\mathbf{0}\} .
$$

Thus $V=\{0\}$ and $X$ is discrete. The rest of (c) (except connectedness) as well as (a) and (b) follow from Proposition 3.1. 
Assume now that $X$ is connected. Consequently, the product spaces $X^{n}$ are also connected, for all $n \in \mathbb{N}$. For $n \in \mathbb{N}$ let

$$
G_{n}:=\left\{\mathbf{x} \in c_{0}(X): x_{k}=0, k=n+1, n+2, \ldots\right\} .
$$

Then $G_{n}$ is a subgroup of $c_{0}(X)$ and $\left(G_{n}, \mathfrak{u}_{\mid G_{n}}\right)$ is topologically isomorphic to $\left(X^{n}, \mathfrak{p}_{\mid X^{n}}\right)$, therefore connected. Since $X^{(\mathbb{N})}=\bigcup_{n \in \mathbb{N}} G_{n}$ and $\bigcap_{n \in \mathbb{N}} G_{n} \neq \emptyset$, we obtain that $\left(X^{(\mathbb{N})}, \mathfrak{u}_{\mid X^{(\mathbb{N})}}\right)$ and its closure $\left(c_{0}(X), \mathfrak{u}_{0}\right)$ are connected as well.

Remark 3.5. As already anticipated in the introduction, the assignment (1.1) defines a functor $\mathcal{F}: \mathfrak{H} \rightarrow \mathfrak{H}$, where $\mathfrak{S}$ is the category of Hausdorff topological abelian groups and continuous homomorphisms. A more precise definition is given in item (a) below.

(a) $\mathcal{F} X:=\left(c_{0}(X), \mathfrak{u}_{0}\right)$ for every $X \in \mathfrak{H}$. For every morphism $f: X \rightarrow Y$ in $\mathfrak{H}$ and every $\left(x_{n}\right) \in c_{0}(X)$, the sequence $\left(f\left(x_{n}\right)\right)$ is a null sequence in $Y$, therefore it defines a group homomorphism $\mathfrak{F}(f): c_{0}(X) \rightarrow c_{0}(Y)$. It is easy to check that $\mathfrak{F}(f)$ is $\mathfrak{u}_{0}$-continuous, thus $\mathfrak{F}(f): \mathfrak{F} X \rightarrow \mathfrak{F} Y$ is a morphism in $\mathfrak{5}$.

(b) According to item (c) of the above proposition, the endofunctor $\mathcal{F}: \mathfrak{H} \rightarrow \mathfrak{H}$ preserves the full subcategories LQC and MAP, as well as all other full subcategories determined by the properties listed there. In particular, $\mathfrak{F}$ defines an endofunctor of the full subcategory of all (connected) Polish LQC groups.

(c) According to Proposition $3.1\left(\mathrm{c}_{2}\right), \mathfrak{F} X$ is precompact only when $X \in \mathfrak{H}$ is the trivial group.

Example 3.6 (The metric group $\left(c_{0}(\mathbb{S}), d_{\infty}\right)$ ). For the particular case $X=\mathbb{S}$, the metric $d_{\infty}$ (defined as in Remark 3.2) generates the topology $\mathfrak{u}_{0}$. This meaningful example was introduced by Rolewicz in [24], where he observed that a complete metrizable monothetic group need not be compact nor discrete. It is well known the dichotomy existing in the class of LCA-groups: namely, a monothetic LCA-group must be either compact or discrete ([30, Lemme 26.2]; see also [1, Remark 5], where a construction of a different example of a complete metrizable monothetic non-locally compact group is indicated).

A proof of the fact that $\left(c_{0}(\mathbb{S}), \mathfrak{u}_{0}\right)$ is monothetic is contained in [15, pp. 20-21] (cf. also [18], where it is shown further that $\left(c_{0}(\mathbb{S}), \mathfrak{u}_{0}\right)$ is Pontryagin reflexive).

Remark 3.7. Let $X$ be the group $\mathbb{R}$ with the usual topology.

(a) By Proposition 3.1 above, $\left(\mathbb{R}^{\mathbb{N}}, \mathfrak{u}\right)$ is a complete metrizable topological abelian group. The group $\left(\mathbb{R}^{\mathbb{N}}, u\right)$ is not connected; the connected component of the null element coincides with $l_{\infty}$ and the topology $\left.\mathfrak{u}\right|_{l_{\infty}}$ is the usual Banach- 
space topology of $l_{\infty}$. It follows that although $\mathbb{R}^{\mathbb{N}}$ is a vector space over $\mathbb{R}$, the topological group $\left(\mathbb{R}^{\mathbb{N}}, \mathfrak{u}\right)$ is not a topological vector space over $\mathbb{R}$.

(b) By Proposition $3.4(c),\left(c_{0}(\mathbb{R}), \mathfrak{u}_{0}\right)$ is a complete separable metrizable connected topological abelian group. Note that $c_{0}(\mathbb{R})$ is a vector space over $\mathbb{R}$ and $\left(c_{0}(\mathbb{R}), \mathfrak{u}_{0}\right)$ is a topological vector space over $\mathbb{R}$. The topology $\mathfrak{u}_{0}$ is the usual Banach-space topology of $c_{0}$.

(c) It is easy to see that $\mathbb{Z}^{(\mathbb{N})}$ is a closed subgroup of $\left(c_{0}(\mathbb{R}), \mathfrak{u}_{0}\right)$ and the quotient group

$$
\left(c_{0}(\mathbb{R}), \mathfrak{u}_{0}\right) / \mathbb{Z}^{(\mathbb{N})}
$$

is topologically isomorphic with $\left(c_{0}(\mathbb{S}), \mathfrak{u}_{0}\right)$.

For an additive topological abelian group $X$ we introduce the following three subgroups of $X^{\mathbb{N}}$ placed between $X^{(\mathbb{N})}$ and $c_{0}(X)$ :

$$
\begin{aligned}
& \operatorname{cs}(X)=\left\{\mathbf{x}=\left(x_{n}\right)_{n \in \mathbb{N}} \in X^{\mathbb{N}}:\left(\sum_{k=1}^{n} x_{k}\right)_{n \in \mathbb{N}} \text { is a Cauchy sequence in } X\right\}, \\
& \operatorname{ss}(X)=\left\{\mathbf{x}=\left(x_{n}\right)_{n \in \mathbb{N}} \in X^{\mathbb{N}}:\left(\sum_{k=1}^{n} x_{k}\right)_{n \in \mathbb{N}} \text { is a convergent sequence in } X\right\}
\end{aligned}
$$

and

$$
\begin{aligned}
l(X)=\left\{\mathbf{x}=\left(x_{n}\right)_{n \in \mathbb{N}} \in X^{\mathbb{N}}:\right. & \left(x_{\sigma(n)}\right)_{n \in \mathbb{N}} \in \operatorname{ss}(X) \\
& \text { for every bijection } \sigma: \mathbb{N} \rightarrow \mathbb{N}\} .
\end{aligned}
$$

The same notation will be used if $X$ is a multiplicative topological abelian group.

Clearly,

$$
X^{(\mathbb{N})} \subset l(X) \subset \operatorname{ss}(X) \subset \operatorname{cs}(X) \subset c_{0}(X) .
$$

It is easy to observe that for a Hausdorff topological abelian group $X$ the equality $\operatorname{ss}(\mathrm{X})=\operatorname{cs}(\mathrm{X})$ holds iff $X$ is sequentially complete.

The symbols $\operatorname{cs}(X)$ and $\operatorname{ss}(X)$ are not standard, while $l(X)$ can be justified as follows: usually $l$ stands for the set of all real absolutely summable sequences and by the Riemann-Dirichlet Theorem,

$$
l(\mathbb{R})=\left\{\mathbf{x}=\left(x_{n}\right)_{n \in \mathbb{N}} \in \mathbb{R}^{\mathbb{N}}:\left(\left|x_{n}\right|\right)_{n \in \mathbb{N}} \in \operatorname{ss}(\mathbb{R})\right\}=l .
$$

Observe that we also have the following analogue of (3.3) for $\mathbb{S}$ (cf. [9, Chapter VIII.2, Theorem 1, p. 116]):

$$
l(\mathbb{S})=\left\{\mathbf{x}=\left(x_{n}\right)_{n \in \mathbb{N}} \in \mathbb{S}^{\mathbb{N}}:\left(\left|1-x_{n}\right|\right)_{n \in \mathbb{N}} \in \operatorname{ss}(\mathbb{R})\right\} .
$$

It is well known that $l(\mathbb{R}) \neq \operatorname{ss}(\mathbb{R}) \neq \mathrm{c}_{0}(\mathbb{R})$. 
Let us consider now the situation in the general case. First of all we note that if $X$ has only trivial convergent sequences, then

$$
X^{(\mathbb{N})}=l(X)=\operatorname{ss}(X)=\operatorname{cs}(X)=c_{0}(X) .
$$

However, for a group $X$ the equality $\operatorname{cs}(\mathrm{X})=\mathrm{c}_{0}(\mathrm{X})$ need not imply the equality $X^{(\mathbb{N})}=c_{0}(X)$ as the following proposition shows.

Proposition 3.8. Let $X$ be a topological abelian group.

(a) If $X$ has a linear topology, then $\operatorname{cs}(X)=c_{0}(X)$.

(b) If $X$ is sequentially complete and $\mathcal{N}(X)$ admits a base consisting of subgroups of $X$, then $l(X)=\operatorname{ss}(X)=c_{0}(X)$.

(c) If $X$ is totally disconnected and locally compact, then $l(X)=\operatorname{ss}(X)=c_{0}(X)$.

Proof. Statement (a) is easy to verify and (b) follows from (a). Finally, statement (c) follows from (b), since our hypotheses imply that $X$ has a linear topology ([20, Theorem II.7.7, p. 62]).

We shall see (Remark 6.16) that if a non-trivial group $X$ is either connected and metrizable or connected and locally compact, then $\operatorname{ss}(X) \neq c_{0}(X)$. It is not clear whether for a complete metrizable abelian group $X$ the equality $l(X)=\operatorname{ss}(X)$ implies the equality $\operatorname{ss}(X)=c_{0}(X)$.

\section{The $\boldsymbol{\beta}$-dual of a group of sequences}

For topological abelian groups $X, Y$ and a non-empty $A \subset X^{\mathbb{N}}$ we write

$$
\begin{aligned}
A^{\beta}(Y)=\left\{\mathbf{h}=\left(\xi_{n}\right)_{n \in \mathbb{N}} \in(\operatorname{CHom}(X, Y))^{\mathbb{N}}:\right. & \\
& \left.\left(\xi_{n}\left(x_{n}\right)\right)_{n \in \mathbb{N}} \in \operatorname{ss}(Y), \quad \forall \mathbf{x}=\left(x_{n}\right)_{n \in \mathbb{N}} \in A\right\} .
\end{aligned}
$$

The notation $A^{\beta}(Y)$ is taken from the theory of sequence spaces, where it is used for $X=Y=\mathbb{R}$ (see, e.g., [6]).

Instead of $A^{\beta}(\mathbb{S})$ we will use the shorter notation $A^{\beta}$. In other words,

$$
A^{\beta}=\left\{\mathbf{h}=\left(\xi_{n}\right)_{n \in \mathbb{N}} \in\left(X^{\wedge}\right)^{\mathbb{N}}:\left(\xi_{n}\left(x_{n}\right)\right)_{n \in \mathbb{N}} \in \operatorname{ss}(\mathbb{S}), \quad \forall \mathbf{x}=\left(x_{n}\right)_{n \in \mathbb{N}} \in A\right\} .
$$

Clearly, $A^{\beta}$ is a subgroup of $\left(X^{\wedge}\right)^{\mathbb{N}}$ containing $\left(X^{\wedge}\right)^{(\mathbb{N})}$. If $A$ is a subgroup, we will call $A^{\beta}$ the $\beta$-dual of $A$.

Remark 4.1. (a) The definition of the $\beta$-dual of a subgroup $A$ of $X^{\mathbb{N}}$ makes no recourse to a specific topology on $A$. The topology of $X$ plays some role since the elements of $A^{\beta}$ are in $\left(X^{\wedge}\right)^{\mathbb{N}}$. 
(b) Suppose now that $A \subset X^{\mathbb{N}}$ is a subgroup and $\tau$ a group topology in $A$. The main motivation to introduce the $\beta$-dual $A^{\beta}$ comes from the fact that, under appropriate hypotheses, $(A, \tau)^{\wedge}$ is canonically isomorphic with $A^{\beta}$. We describe several cases when this occurs, being $A:=c_{0}(X)$ the most important of them (see Proposition 4.4).

We calculate now the $\beta$-duals of the most natural groups of sequences, $X^{(\mathbb{N})}$ and $X^{\mathbb{N}}$.

Lemma 4.2. Let $X$ be a topological abelian group. Then

$$
\left(X^{(\mathbb{N})}\right)^{\beta}=\left(X^{\wedge}\right)^{\mathbb{N}} \text { and }\left(X^{\mathbb{N}}\right)^{\beta}=\left(X^{\wedge}\right)^{(\mathbb{N})} .
$$

Proof. The first equality in (4.1) is trivial. To prove the second one, it is sufficient to show that $\left(X^{\mathbb{N}}\right)^{\beta} \subset\left(X^{\wedge}\right)^{(\mathbb{N})}$. Take

$$
\mathbf{h}=\left(\xi_{n}\right)_{n \in \mathbb{N}} \in\left(X^{\mathbb{N}}\right)^{\beta}
$$

and let us see that the assumption

$$
\mathbf{h}=\left(\xi_{n}\right)_{n \in \mathbb{N}} \notin\left(X^{\wedge}\right)^{(\mathbb{N})}
$$

leads to a contradiction with (4.2).

From (4.3) we get the existence of a strictly increasing sequence $\left(k_{n}\right)_{n \in \mathbb{N}}$ of natural numbers such that

$$
\xi_{k_{n}}(X) \neq\{1\}, \quad n=1,2, \ldots
$$

Since $\mathbb{S}_{+}$does not contain nontrivial subgroups of $\mathbb{S}$, (4.4) provides a sequence $\left(x_{k_{n}}\right)_{n \in \mathbb{N}}$ of elements of $X$ such that

$$
\xi_{k_{n}}\left(x_{k_{n}}\right) \notin \mathbb{S}_{+}, \quad n=1,2, \ldots
$$

Putting $x_{j}=0$ for all $j \in \mathbb{N} \backslash\left\{k_{1}, k_{2}, \ldots\right\}$, we get a sequence $\mathbf{x}=\left(x_{n}\right)_{n \in \mathbb{N}} \in X^{\mathbb{N}}$ such that $\left(\prod_{k=1}^{n} \xi_{k}\left(x_{k}\right)\right)_{n \in \mathbb{N}}$ converges in $\mathbb{S}$ by virtue of (4.2). Then

$$
\lim _{n} \xi_{n}\left(x_{n}\right)=1 \text {, }
$$

so $\lim _{n} \xi_{k_{n}}\left(x_{k_{n}}\right)=1$. This contradicts (4.5), as $\mathbb{S}_{+} \in \mathcal{N}(\mathbb{S})$.

Aiming to calculate the $\beta$-dual of the more complicated group of sequences $c_{0}(X)$, we need the following:

Lemma 4.3. Let $X$ be a topological abelian group. Then $c_{0}(X)^{\beta}$ is stable under taking subsequences.

Proof. Let $\left(\xi_{n}\right)_{n \in \mathbb{N}} \in c_{0}(X)^{\beta}$ and $\left(k_{n}\right)_{n \in \mathbb{N}}$ be a strictly increasing sequence of natural numbers. We have to check that $\left(\xi_{k_{n}}\right)_{n \in \mathbb{N}} \in c_{0}(X)^{\beta}$. To this end, take an 
arbitrary sequence $\mathbf{x}=\left(x_{n}\right)_{n \in \mathbb{N}} \in c_{0}(X)$ and define $\mathbf{y}=\left(y_{n}\right)_{n \in \mathbb{N}} \in X^{\mathbb{N}}$ as follows: $y_{k_{n}}=x_{n}, n=1,2, \ldots$, and $y_{j}=0, \forall j \in \mathbb{N} \backslash\left\{k_{1}, k_{2}, \ldots\right\}$. Then obviously $\mathbf{y}=\left(y_{n}\right)_{n \in \mathbb{N}} \in c_{0}(X)$. Since $\left(\xi_{n}\right)_{n \in \mathbb{N}} \in c_{0}(X)^{\beta}$, the sequence

$$
\left(\prod_{k=1}^{n} \xi_{k}\left(y_{k}\right)\right)_{n \in \mathbb{N}}
$$

converges in $\mathbb{S}$. Observe that

$$
\prod_{j=1}^{k_{n}} \xi_{j}\left(y_{j}\right)=\prod_{j=1}^{n} \xi_{k_{j}}\left(x_{j}\right), \quad n=1,2, \ldots
$$

From (4.6) we conclude that the sequence

$$
\left(\prod_{j=1}^{n} \xi_{k_{j}}\left(x_{j}\right)\right)_{n \in \mathbb{N}}
$$

converges in $\mathbb{S}$ too. Since this is true for an arbitrary $\mathbf{x}=\left(x_{n}\right)_{n \in \mathbb{N}} \in c_{0}(X)$, we conclude that $\left(\xi_{k_{n}}\right)_{n \in \mathbb{N}} \in c_{0}(X)^{\beta}$.

The following statement is a bit more delicate than Lemma 4.2.

Proposition 4.4. For $c_{0}(\mathbb{S})$ we have

$$
c_{0}(\mathbb{S})^{\beta}=\left(\mathbb{S}^{\wedge}\right)^{(\mathbb{N})} .
$$

Proof. For a fixed $m \in \mathbb{Z}$ let $\varphi_{m}: \mathbb{S} \rightarrow \mathbb{S}$ be the mapping $t \mapsto t^{m}$. It is known that

$$
\mathbb{S}^{\wedge}=\left\{\varphi_{m}: m \in \mathbb{Z}\right\}
$$

So, fix a sequence $\left(m_{n}\right)_{n \in \mathbb{N}} \in \mathbb{Z}^{\mathbb{N}}$ such that $\left(\varphi_{m_{n}}\right)_{n \in \mathbb{N}} \in c_{0}(\mathbb{S})^{\beta}$ and let us see that in fact $\left(m_{n}\right)_{n \in \mathbb{N}} \in \mathbb{Z}^{(\mathbb{N})}$.

Suppose that we have $\left(m_{n}\right)_{n \in \mathbb{N}} \notin \mathbb{Z}^{(\mathbb{N})}$. Then for some strictly increasing sequence $\left(k_{n}\right)_{n \in \mathbb{N}}$ of natural numbers we shall have $m_{k_{n}} \neq 0, n=1,2, \ldots$ Since $\left(\varphi_{m_{n}}\right)_{n \in \mathbb{N}} \in c_{0}(\mathbb{S})^{\beta}$, by Lemma 4.3 we have

$$
\left(\varphi_{m_{k n}}\right)_{n \in \mathbb{N}} \in c_{0}(\mathbb{S})^{\beta} .
$$

Let $x_{1}=x_{2}=1$; then for a natural number $j>2$ find the unique natural number $n$ with $2^{n}<j \leq 2^{n+1}$ and write

$$
x_{j}=\exp \left(2 \pi i \frac{1}{m_{k_{j}} 2^{n+1}}\right) .
$$


Clearly, $\mathbf{x}=\left(x_{j}\right)_{j \in \mathbb{N}} \in c_{0}(\mathbb{S})$ and

$$
\prod_{j=2^{n}+1}^{2^{n+1}} \varphi_{m_{k_{j}}}\left(x_{j}\right)=\exp \left(2 \pi i \sum_{k=2^{n}+1}^{2^{n+1}} \frac{1}{2^{n+1}}\right)=-1, \quad n=1,2, \ldots
$$

It follows from (4.9) that

$$
\left(\prod_{j=1}^{n} \varphi_{m_{k_{j}}}\left(x_{j}\right)\right)_{n \in \mathbb{N}}
$$

is not a Cauchy sequence in $\mathbb{S}$, hence it is not convergent in $\mathbb{S}$ in contradiction with equation (4.8).

\section{The $\beta$-dual related to the algebraic dual}

For a topological abelian group $X$, a subgroup $A \subset X^{\mathbb{N}}$ and for a fixed sequence $\mathbf{h}=\left(\xi_{n}\right)_{n \in \mathbb{N}} \in A^{\beta}$ we define a mapping $\chi_{\mathbf{h}}: A \rightarrow \mathbb{S}$ by the equality

$$
\chi_{\mathbf{h}}(\mathbf{x})=\prod_{n=1}^{\infty} \xi_{n}\left(x_{n}\right):=\lim _{n} \prod_{k=1}^{n} \xi_{k}\left(x_{k}\right), \quad \mathbf{x}=\left(x_{n}\right)_{n \in \mathbb{N}} \in A .
$$

It is easy to observe that

(1) if $\mathbf{h}=\left(\xi_{n}\right)_{n \in \mathbb{N}} \in\left(X^{\wedge}\right)^{(\mathbb{N})}$, then $\chi_{\mathbf{h}}$ is defined on the whole $X^{\mathbb{N}}$,

(2) for every subgroup $A$ of $X^{\mathbb{N}}$,

$$
\chi_{\mathbf{h}} \in \operatorname{Hom}(A, \mathbb{S}), \quad \forall \mathbf{h} \in A^{\beta} .
$$

Notation 4.5. For a subgroup $A \subset X^{\mathbb{N}}$, the letter $\chi$ will denote in the sequel the mapping

$$
\chi: A^{\beta} \rightarrow \operatorname{Hom}(A, \mathbb{S})
$$

defined by the equality

$$
\chi(\mathbf{h})=\chi_{\mathbf{h}}, \quad \forall \mathbf{h}=\left(\xi_{n}\right)_{n \in \mathbb{N}} \in A^{\beta} .
$$

Although the mapping $\chi$ depends on $A$, in order to simplify notation we do not indicate this dependence.

The following lemma relates the algebraic dual and the $\beta$-dual of a subgroup $A$ of $X^{\mathbb{N}}$.

Lemma 4.6. Let $X$ be a topological abelian group and $A \subset X^{\mathbb{N}}$ a subgroup such that $X^{(\mathbb{N})} \subset A$. Then, the mapping $\chi: A^{\beta} \rightarrow \operatorname{Hom}(A, \mathbb{S})$ is an injective group homomorphism. 
Proof. It is easy to see that the considered mapping $\chi$ is a group homomorphism. Let $\mathbf{h}=\left(\xi_{n}\right)_{n \in \mathbb{N}} \in \operatorname{ker}(\chi)$. Then

$$
\chi_{\mathbf{h}}(\mathbf{x})=1, \quad \forall \mathbf{x}=\left(x_{n}\right)_{n \in \mathbb{N}} \in A .
$$

Fix $n \in \mathbb{N}$ and $x \in X$. As $v_{n}(x) \in X^{(\mathbb{N})} \subset A$, we get $\xi_{n}(x)=\chi_{\mathbf{h}}\left(v_{n}(x)\right)=1$. Since $x \in X$ is arbitrary, it follows that $\xi_{n}$ must be the null character. Therefore, $\mathbf{h}=1:=(1,1, \ldots)$ and $\operatorname{ker}(\chi)=\{1\}$. Hence, $\chi$ is injective.

The next example illustrates the usefulness of the topology-free Lemma 4.2.

Example 4.7. If $X$ is a topological abelian group, it is well known that

$$
\chi_{\mathbf{h}} \in\left(X^{\mathbb{N}}, \mathfrak{p}\right)^{\wedge}, \quad \forall \mathbf{h}=\left(\xi_{n}\right)_{n \in \mathbb{N}} \in\left(X^{\wedge}\right)^{(\mathbb{N})}=\left(X^{\mathbb{N}}\right)^{\beta},
$$

and the mapping $\chi:\left(X^{\wedge}\right)^{(\mathbb{N})} \rightarrow\left(X^{\mathbb{N}}, \mathfrak{p}\right)^{\wedge}$ is a group isomorphism.

Indeed, (4.10) is easy to verify. The injectivity of $\chi$ derives from Lemma 4.6. It remains to show that $\chi$ is surjective too.

Write $G=\left(X^{\mathbb{N}}, \mathfrak{p}\right)$ and fix an arbitrary $\kappa \in G^{\wedge}$. We need to find a sequence $\mathbf{h}=\left(\xi_{n}\right)_{n \in \mathbb{N}} \in\left(X^{\wedge}\right)^{(\mathbb{N})}$ such that $\kappa=\chi_{\mathbf{h}}$. For every $n \in \mathbb{N}$ the homomorphism $\nu_{n}: X \rightarrow G$ is continuous. Hence we obtain that $\xi_{n}:=\kappa \circ v_{n} \in X^{\wedge}$. Let us see that $\mathbf{h}:=\left(\xi_{n}\right)_{n \in \mathbb{N}}$ meets the requirements.

Fix $\mathbf{x}=\left(x_{n}\right)_{n \in \mathbb{N}} \in X^{\mathbb{N}}$. Evidently, the sequence $\left(\sum_{k=1}^{n} v_{k}\left(x_{k}\right)\right)_{n \in \mathbb{N}}$ converges in $G$ to $\mathbf{x}=\left(x_{n}\right)_{n \in \mathbb{N}}$. Hence

$$
\kappa(\mathbf{x})=\lim _{n} \kappa\left(\sum_{k=1}^{n} v_{k}\left(x_{k}\right)\right)=\lim _{n} \prod_{k=1}^{n} \xi_{k}\left(x_{k}\right) .
$$

Since $\mathbf{x}=\left(x_{n}\right)_{n \in \mathbb{N}} \in X^{\mathbb{N}}$ is arbitrary, (4.11) implies that $\mathbf{h}=\left(\xi_{n}\right)_{n \in \mathbb{N}} \in\left(X^{\mathbb{N}}\right)^{\beta}$ and $\kappa=\chi_{\mathbf{h}}$. By Lemma 4.2, we have $\left(X^{\mathbb{N}}\right)^{\beta}=\left(X^{\wedge}\right)^{(\mathbb{N})}$. Consequently we have found $\mathbf{h}=\left(\xi_{n}\right)_{n \in \mathbb{N}} \in\left(X^{\wedge}\right)^{(\mathbb{N})}$ such that $\kappa=\chi_{\mathbf{h}}$, and the surjectivity of $\chi$ is thus proved.

Remark 4.8. Let $X$ be a topological abelian group.

(a) Example 4.7 asserts only that the group $\left(X^{\mathbb{N}}, \mathfrak{p}\right)^{\wedge}$ can be algebraically identified with the group $\left(X^{\wedge}\right)^{(\mathbb{N})}$ by means of the group isomorphism $\chi$. In fact more is known: the mapping $\chi$ is also a homeomorphism between $\left(X^{\mathbb{N}}, \mathfrak{p}\right)_{\mathrm{co}}^{\wedge}$ and $\left(\left(X^{\wedge}\right)^{(\mathbb{N})}, \hat{\mathfrak{b}}\right)$, where $\hat{\mathfrak{b}}$ stands for the topology induced from the box product $\left(\left(X_{\mathrm{co}}^{\wedge}\right)^{\mathbb{N}}, \mathfrak{b}\right)$ in $\left(X^{\wedge}\right)^{(\mathbb{N})}$.

(b) An application of (a) for $X=\mathbb{Z}$ gives that $\left(\mathbb{S}^{(\mathbb{N})},\left.\mathfrak{b}\right|_{\mathbb{S}(\mathbb{N})}\right)$ is a complete nonmetrizable group. In particular, we obtain that $\mathbb{S}^{(\mathbb{N})}$ is a closed subgroup of $\left(\mathbb{S}^{\mathbb{N}}, \mathfrak{b}\right)$. 
(c) It is also known that the group $\left(X^{(\mathbb{N})},\left.\mathfrak{b}\right|_{X^{(\mathbb{N})}}\right)_{\text {co }}^{\wedge}$ is topologically isomorphic with $\left(\left(X_{\mathrm{co}}^{\wedge}\right)^{\mathbb{N}}, \mathfrak{p}\right)$.

(d) An application of (c) for $X=\mathbb{S}$ gives that the group $\left(\mathbb{S}^{(\mathbb{N})},\left.\mathfrak{b}\right|_{\mathbb{S}_{(\mathbb{N})}}\right)^{\wedge}$ has cardinality c. It follows that $\left.\mathfrak{b}\right|_{\mathbb{S}(\mathbb{N})}$ is not a compatible topology for $\left(\mathbb{S}^{(\mathbb{N})},\left.\mathfrak{p}\right|_{\mathbb{S}(\mathbb{N})}\right)$ (cf. Proposition 5.2).

A good reference for the topological isomorphisms from (a) and (c) is [21], where the topological duality between sums and product is thoroughly studied.

\section{The case when $A \subset X^{\mathbb{N}}$ is endowed with a group topology: $\beta$-dual versus topological dual.}

So far we have seen that the $\beta$-dual of a group of sequences $A$ can be mapped isomorphically into the algebraic dual $\operatorname{Hom}(A, \mathbb{S})$. If $A$ is further endowed with a topology, then $A^{\beta}$ could be related with the topological dual $A^{\wedge}=\operatorname{CHom}(A, \mathbb{S})$. We are specially interested in the groups of the form $c_{0}(X)$ with the uniform topology and more precisely under which conditions on $X$ the topological dual can be identified with the $\beta$-dual.

In this section we will prove that for a complete metrizable group $X$, the dual of the topological group $\left(c_{0}(X), \mathfrak{u}_{0}\right)$ algebraically coincides with the $\beta$-dual. We already calculated the $\beta$-dual for the particular group $c_{0}(\mathbb{S})$ (Proposition 4.4), which has interest in itself: in fact, from it we derive the first example of a metrizable locally quasi-convex group which is not LQC-Mackey (see Proposition 5.2).

The next proposition, playing a pivotal role in the computation of the dual of $\left(c_{0}(X), \mathfrak{u}_{0}\right)$, clarifies the properties of the injective group homomorphism

$$
\chi: c_{0}(X)^{\beta} \rightarrow \operatorname{Hom}\left(c_{0}(X), \mathbb{S}\right),
$$

defined in Lemma 4.6 in a more general setting.

Proposition 5.1. Let $X$ be a topological abelian group and $G:=\left(c_{0}(X), \mathfrak{u}_{0}\right)$. The following assertions hold:

(a) $\chi\left(\left(X^{\wedge}\right)^{(\mathbb{N})}\right) \subseteq G^{\wedge}$, i.e., $\chi_{\mathbf{h}} \in G^{\wedge}$ for any $\mathbf{h}=\left(\xi_{n}\right)_{n \in \mathbb{N}} \in\left(X^{\wedge}\right)^{(\mathbb{N})} \subset c_{0}(X)^{\beta}$.

(b) $G^{\wedge} \subset \operatorname{Im} \chi=\left\{\chi_{\mathbf{h}}: \mathbf{h}=\left(\xi_{n}\right)_{n \in \mathbb{N}} \in c_{0}(X)^{\beta}\right\}$.

(c) If $X$ is complete metrizable (or, more generally, $\left(c_{0}(X), \mathfrak{u}_{0}\right)$ is a Baire space), then we have

$$
G^{\wedge}=\operatorname{Im} \chi=\left\{\chi_{\mathbf{h}}: \mathbf{h}=\left(\xi_{n}\right)_{n \in \mathbb{N}} \in c_{0}(X)^{\beta}\right\}
$$

and the mapping $\chi: c_{0}(X)^{\beta} \rightarrow G^{\wedge}$ is a group isomorphism. 
Proof. (a) As $\mathfrak{u}_{0} \geq \mathfrak{p}_{0}$, we have $\chi_{\mathbf{h}} \in G^{\wedge}, \forall \mathbf{h}=\left(\xi_{n}\right)_{n \in \mathbb{N}} \in\left(X^{\wedge}\right)^{(\mathbb{N})}$. The rest follows from Lemma 4.6.

(b) Fix $\kappa \in G^{\wedge}$. We need to find $\mathbf{h}=\left(\xi_{n}\right)_{n \in \mathbb{N}} \in c_{0}(X)^{\beta}$ such that $\kappa=\chi_{\mathbf{h}}$. For every $n \in \mathbb{N}$ the homomorphism $v_{n}: X \rightarrow G$ is continuous, so $\xi_{n}:=\kappa \circ v_{n} \in X^{\wedge}$. Let us see that $\mathbf{h}:=\left(\xi_{n}\right)_{n \in \mathbb{N}}$ meets the requirements.

Fix $\mathbf{x}=\left(x_{n}\right)_{n \in \mathbb{N}} \in c_{0}(X)$. By Lemma 3.3 the sequence $\left(\sum_{k=1}^{n} v_{k}\left(x_{k}\right)\right)_{n \in \mathbb{N}}$ converges in $G$ to $\mathbf{x}=\left(x_{n}\right)_{n \in \mathbb{N}}$. Hence

$$
\kappa(\mathbf{x})=\lim _{n} \kappa\left(\sum_{k=1}^{n} v_{k}\left(x_{k}\right)\right)=\lim _{n} \prod_{k=1}^{n} \xi_{k}\left(x_{k}\right) .
$$

As $\mathbf{x}=\left(x_{n}\right)_{n \in \mathbb{N}} \in c_{0}(X)$ is arbitrary, (5.1) implies that $\mathbf{h}=\left(\xi_{n}\right)_{n \in \mathbb{N}} \in c_{0}(X)^{\beta}$ and $\kappa=\chi_{\mathbf{h}}$.

(c) Taking into account (b), we only need to see that $G^{\wedge} \supset \operatorname{Im} \chi$. To this end, fix $\mathbf{h}=\left(\xi_{n}\right)_{n \in \mathbb{N}} \in c_{0}(X)^{\beta}$. As already noted, $\chi_{\mathbf{h}}: c_{0}(X) \rightarrow \mathbb{S}$ is a group homomorphism. For $n \in \mathbb{N}$, set $\mathbf{h}_{n}=\left(\xi_{1}, \ldots, \xi_{n}, 1,1, \ldots\right)$. Then $\mathbf{h}_{n} \in\left(X^{\wedge}\right)^{(\mathbb{N})}$ and hence

$$
\chi_{\mathbf{h}_{n}} \in G^{\wedge}, \quad n=1,2, \ldots
$$

Clearly,

$$
\lim _{n} \chi_{\mathbf{h}_{n}}(\mathbf{x})=\chi_{\mathbf{h}}(\mathbf{x}), \quad \forall \mathbf{x}=\left(x_{n}\right)_{n \in \mathbb{N}} \in c_{0}(X) .
$$

Since $X$ is complete metrizable, by Proposition 3.4 (c), the group $G=\left(c_{0}(X), \mathfrak{u}_{0}\right)$ is complete metrizable too. In particular, $G=\left(c_{0}(X), \mathfrak{u}_{0}\right)$ is a Baire space. This and relations (5.2) and (5.3), according to Osgood's theorem [22, Theorem 9.5] imply that the function $\chi_{\mathbf{h}}$ has a $\mathfrak{u}_{0}$-continuity point $\mathbf{x}=\left(x_{n}\right)_{n \in \mathbb{N}} \in c_{0}(X)$. Since $\chi_{\mathbf{h}}$ is a group homomorphism, we get that $\chi_{\mathbf{h}}$ is $\mathfrak{u}_{\mathbf{0}}$-continuous. Therefore, we obtain $\chi_{\mathbf{h}} \in G^{\wedge}$.

Now we are ready to give the first example of a precompact metrizable group which is not a Mackey group in LQC:

Proposition 5.2. For $X=\mathbb{S}$ the following assertions hold:

(a) $\left(\right.$ cf. $\left[23\right.$, Lemma]) $\left(c_{0}(\mathbb{S}), \mathfrak{p}_{0}\right)^{\wedge}=\left(c_{0}(\mathbb{S}), \mathfrak{u}_{0}\right)^{\wedge}$. In particular, $\left(c_{0}(\mathbb{S}), \mathfrak{u}_{0}\right)^{\wedge}$ is countable.

(b) $\mathfrak{u}_{0}$ is a compatible locally quasi-convex Polish group topology for $\left(c_{0}(\mathbb{S}), \mathfrak{p}_{0}\right)$. Further, $\left(c_{0}(\mathbb{S}), u_{0}\right)$ is connected and monothetic.

(c) $\left(c_{0}(\mathbb{S}), p_{0}\right)$ is a precompact metrizable group which is not a Mackey group in LQC.

Proof. (a) Since $\mathfrak{p}_{0} \leq \mathfrak{u}$, we have $\left(c_{0}(\mathbb{S}), \mathfrak{p}_{0}\right)^{\wedge} \subset\left(c_{0}(\mathbb{S}), \mathfrak{u}_{0}\right)^{\wedge}$. To prove the converse inclusion, fix an arbitrary $\kappa \in\left(c_{0}(\mathbb{S}), \mathfrak{u}_{0}\right)^{\wedge}$. By Proposition $5.1(\mathrm{~b})$, there is 
a sequence $\mathbf{h}=\left(\xi_{n}\right)_{n \in \mathbb{N}} \in\left(c_{0}(\mathbb{S})\right)^{\beta}$ such that $\kappa=\chi_{\mathbf{h}}$. By Proposition 4.4,

$$
c_{0}(\mathbb{S})^{\beta}=\left(\mathbb{S}^{\wedge}\right)^{(\mathbb{N})} \text {. }
$$

Therefore we have $\kappa=\chi_{\mathbf{h}}$, where $\mathbf{h}=\left(\xi_{n}\right)_{n \in \mathbb{N}} \in\left(\mathbb{S}^{\wedge}\right)^{(\mathbb{N})}$. Consequently, we get $\kappa \in\left(c_{0}(\mathbb{S}), p_{0}\right)^{\wedge}$ and the first part of (a) is proved. The second part of (a) follows from the first one because $\left(c_{0}(\mathbb{S}), \mathfrak{p}_{0}\right)^{\wedge}$ is algebraically isomorphic to $\left(\mathbb{S}^{\mathbb{N}}, \mathfrak{p}\right)^{\wedge}$.

(b) By Proposition 3.4, $\mathfrak{u}_{0}$ is a locally quasi-convex Polish group topology. It is compatible for $\left(c_{0}(\mathbb{S}), p_{0}\right)$ by (a). The last two assertions are respectively contained in Proposition 3.4 (c) and Example 3.6.

(c) Observe that $\left(c_{0}(\mathbb{S}), p_{0}\right)$ is a topological subgroup of the compact metrizable group $\left(\mathbb{S}^{\mathbb{N}}, \mathfrak{p}\right)$. Therefore it is metrizable and precompact. By $(b), \mathfrak{u}_{0}$ is a locally quasi-convex group topology compatible for $\left(c_{0}(\mathbb{S}), \mathfrak{p}_{0}\right)$ and strictly finer than $p_{0}$ (by Proposition 3.4). This finally proves that $\left(c_{0}(\mathbb{S}), \mathfrak{p}_{0}\right)$ is not a Mackey group in LQC.

Remark 5.3. It follows from Propositions 3.4 and $5.2(a)$ that $\left(c_{0}(\mathbb{S}), \mathfrak{u}_{0}\right)$ is a nonprecompact locally quasi-convex group with countable dual $\left(c_{0}(\mathbb{S}), \mathfrak{u}_{0}\right)^{\wedge}$; consequently by Theorem $1.8\left(c_{0}(\mathbb{S}),\left(\mathfrak{u}_{0}\right)^{+}\right)$is a metrizable precompact group which is not a Mackey group in LQC. Observe that (again by Proposition 5.2 (a)) we have $\left(\mathfrak{u}_{0}\right)^{+}=p_{0}$ and we get a second proof of Proposition $5.2(\mathrm{c})$.

We shall see below that the group $\mathbb{S}$ in Proposition 5.2 can be replaced by an arbitrary non-trivial compact connected metrizable group (see Theorem 7.3). However the proof of this fact will require a careful preparation, to which the rest of the paper is devoted.

\section{The class $\mathfrak{B}$}

In this section we introduce a large class of compact metrizable groups $X$ that can be used as input in Proposition 5.2.

Definition 6.1. For a topological abelian group $X$, let

$$
\Gamma_{\text {abs }}(X):=\left\{\xi \in X^{\wedge}:\left(\xi\left(x_{n}\right)\right)_{n \in \mathbb{N}} \in \operatorname{ss}(\mathbb{S}), \quad \forall \mathbf{x}=\left(\mathrm{x}_{\mathrm{n}}\right)_{\mathrm{n} \in \mathbb{N}} \in \mathrm{c}_{0}(\mathrm{X})\right\} .
$$

Clearly, $\Gamma_{\mathrm{abs}}(X)$ is a subgroup of $X^{\wedge}$. Denote by $\mathfrak{B}$ the class of groups $X$ such that $\Gamma_{\text {abs }}(X)=\{1\}$.

Remark 6.2. Let $X$ be a topological abelian group.

(a) The notation $\Gamma_{\mathrm{abs}}(X)$ is justified by the following facts easy to prove:

$$
\Gamma_{\text {abs }}(X)=\left\{\xi \in X^{\wedge}:\left(\xi\left(x_{n}\right)\right)_{n \in \mathbb{N}} \in l(\mathbb{S}), \quad \forall \mathbf{x}=\left(x_{n}\right)_{n \in \mathbb{N}} \in c_{0}(X)\right\} .
$$


Taking into account the equality (3.4), for any character $\xi \in X^{\wedge}$, we have

$$
\xi \in \Gamma_{\mathrm{abs}}(X) \text { iff } \sum_{k=1}^{\infty}\left|1-\xi\left(x_{k}\right)\right|<\infty, \forall \mathbf{x}=\left(x_{n}\right)_{n \in \mathbb{N}} \in c_{0}(X) .
$$

(b) $\Gamma_{\text {abs }}(X)$ can be described also by means of the diagonal homomorphism

$$
\Delta: X^{\wedge} \longrightarrow\left(X^{\wedge}\right)^{\mathbb{N}} \text {. }
$$

Clearly, if $\xi \in X^{\wedge}$, then $\xi \in \Gamma_{\text {abs }}(X)$ iff $(\xi, \xi, \ldots, \xi, \ldots) \in c_{0}(X)^{\beta}$. Therefore

$$
\Gamma_{\text {abs }}(X)=\Delta^{-1}\left(c_{0}(X)^{\beta}\right) .
$$

Whenever $c_{0}(X)^{\beta}=\left(X^{\wedge}\right)^{(\mathbb{N})}, \Gamma_{\text {abs }}(X)=\{1\}$, and $X \in \mathfrak{B}$. In particular, by Proposition $4.4, \mathbb{S} \in \mathfrak{B}$.

(c) The introduction of the class $\mathfrak{B}$ is motivated by our ultimate aim to have the equality $c_{0}(X)^{\wedge}=\left(X^{\wedge}\right)^{(\mathbb{N})}$. More precisely, we show below (Theorem 6.3) that $X \in \mathfrak{B}$ for a precompact group iff $\left(c_{0}(X), \mathfrak{u}_{0}\right)^{\wedge}$ can be canonically identified with $\left(X^{\wedge}\right)^{(\mathbb{N})}$. Thus, summarizing:

$$
c_{0}(X)^{\beta}=\left(X^{\wedge}\right)^{(\mathbb{N})} \Longrightarrow X \in \mathfrak{B},
$$

whilst

$$
X \in \mathfrak{B} \& X \text { is precompact } \Longrightarrow c_{0}(X)^{\wedge}=\left(X^{\wedge}\right)^{(\mathbb{N})} .
$$

(d) If $X$ admits a structure of a topological vector space over $\mathbb{R}$, then it is easy to see that $X \in \mathfrak{B}$.

In what follows assume that $c_{0}(X)$ is endowed with the topology $\mathfrak{u}_{0}$. The dual $c_{0}(X)^{\wedge}$ is a subgroup of the group $\operatorname{Hom}\left(c_{0}(X), \mathbb{S}\right)$, and the density of $X^{(\mathbb{N})}$ in $c_{0}(X)$ allows the algebraic identification of $c_{0}(X)^{\wedge}$ with a subgroup of $\left(X^{\wedge}\right)^{\mathbb{N}}$ contained in $c_{0}(X)^{\beta}$ (see also the assignment $\kappa \mapsto\left(\xi_{n}\right)$ from the proof of item (b) of Proposition 5.1). In the sequel we denote by $j: G^{\wedge} \rightarrow\left(X^{\wedge}\right)^{\mathbb{N}}$ the assignment $\kappa \mapsto\left(\xi_{n}\right)$, which actually is the inverse of $\chi$ defined in the previous section. Then $j\left(G^{\wedge}\right) \subseteq c_{0}(X)^{\beta}$.

Theorem 6.3. If $X \in \mathfrak{B}$ is precompact and $G:=\left(c_{0}(X), \mathfrak{u}_{0}\right)$, then

$$
j\left(G^{\wedge}\right)=\left(X^{\wedge}\right)^{(\mathbb{N})} .
$$

Hence the mapping $\chi:\left(X^{\wedge}\right)^{(\mathbb{N})} \rightarrow G^{\wedge}$ is a group isomorphism.

Proof. By Proposition 5.1(a), we only need to see that $\chi$ is surjective. To this end, fix

$$
\mathbf{h}=\left(\xi_{n}\right)_{n \in \mathbb{N}} \in j\left(G^{\wedge}\right) \subseteq c_{0}(X)^{\beta} \subseteq\left(X^{\wedge}\right)^{\mathbb{N}} .
$$

We have to see that, in fact, $\mathbf{h} \in\left(X^{\wedge}\right)^{(\mathbb{N})}$. 
By using the $\mathfrak{u}_{0}$-continuity of $\chi_{\mathbf{h}}$, we can find some $V \in \mathcal{N}(X)$ such that

$$
\chi_{\mathbf{h}}\left(V^{\mathbb{N}} \cap c_{0}(X)\right) \subset \mathbb{S}_{+} .
$$

This implies

$$
\xi_{n} \in V^{\triangleright}, \quad \forall n \in \mathbb{N} .
$$

By Proposition 2.2, the precompactness of $X$ implies that the set $V^{\triangleright}$ is finite. For $\xi \in V^{\triangleright}$ let

$$
\mathbb{N}_{\xi}:=\left\{n \in \mathbb{N}: \xi=\xi_{n}\right\} .
$$

Let us prove that if $\mathbb{N}_{\xi}$ is finite for every $\xi \in V^{\triangleright}$, we have $\xi \neq 1$. Indeed, assume that $\mathbb{N}_{\xi}$ is infinite for some $\xi \in V^{\triangleright}$. Write $\mathbb{N}_{\xi}$ as a strictly increasing sequence: $\mathbb{N}_{\xi}=\left\{k_{1}, k_{2}, \ldots\right\}$. From the fact that $\left(\xi_{n}\right)_{n \in \mathbb{N}} \in c_{0}(X)^{\beta}$ by Lemma 4.3 we conclude that $\left(\xi_{k_{n}}\right)_{n \in \mathbb{N}} \in c_{0}(X)^{\beta}$. Hence we have $(\xi, \xi, \ldots, \xi, \ldots) \in c_{0}(X)^{\beta}$, and so $\xi \in \Gamma_{\text {abs }}(X)$. As $X \in \mathfrak{B}$, we obtain that $\xi=1$. This proves our claim.

As the set $V^{\triangleright}$ is finite and since the above claim implies that each $1 \neq \xi \in V^{\triangleright}$ may appear only finitely many times as a coordinate $\xi_{n}=\xi$ of $\mathbf{h}$, we deduce that for some $n_{0} \in \mathbb{N}$ we have $\xi_{n}=1, \forall n \geq n_{0}$. Consequently,

$$
\mathbf{h}=\left(\xi_{n}\right)_{n \in \mathbb{N}} \in\left(X^{\wedge}\right)^{(\mathbb{N})}
$$

and the surjectivity of $\chi$ is proved.

Corollary 6.4. If $X \in \mathfrak{B}$ is a non-trivial precompact group and $G:=\left(c_{0}(X), \mathfrak{u}_{0}\right)$, then $\left|G^{\wedge}\right|=\left|X^{\wedge}\right|$.

Proof. Any nontrivial precompact group $X \in \mathfrak{B}$ is infinite. Indeed, if $X$ were finite, then $c_{0}(X)$ would coincide with $X^{(\mathbb{N})}$. But then $\Gamma_{\text {abs }}(X) \neq 1$, which contradicts the fact that $X \in \mathfrak{B}$. Thus, $X^{\wedge}$ is infinite as well. According to Theorem 6.3, $\left(X^{\wedge}\right)^{(\mathbb{N})} \cong G^{\wedge}$. Hence, $\left|G^{\wedge}\right|=\left|\left(X^{\wedge}\right)^{(\mathbb{N})}\right|=\left|X^{\wedge}\right|$.

\section{Properties of the class $\mathfrak{B}$}

Proposition 6.5. Let $X$ be a topological abelian group.

(a) If either $\operatorname{cs}(\mathrm{X})=\mathrm{c}_{0}(\mathrm{X})$ or $X$ has no non-trivial convergent sequences, then $\Gamma_{\mathrm{abs}}(X)=X^{\wedge}$.

(b) If $\operatorname{cs}(\mathrm{X})=\mathrm{c}_{0}(\mathrm{X})$ and $X^{\wedge} \neq\{1\}$, then $X \notin \mathfrak{B}$.

(c) If $X$ has a linear topology, then $X \in \mathfrak{B}$ if and only if $X$ is indiscrete.

(d) If $X \neq\{0\}$ is locally compact and totally disconnected, then $X \notin \mathfrak{B}$.

(e) If $X$ has no non-trivial convergent sequences and $X \in \mathfrak{B}$, then $X$ is minimally almost periodic. 
Proof. Statement (a) is easy to verify and (b) follows from (a).

(c) By Proposition $3.8(\mathrm{a}), \operatorname{cs}(\mathrm{X})=\mathrm{c}_{0}(\mathrm{X})$, so item (b), along with $X \in \mathfrak{B}$, implies $X^{\wedge}=\{1\}$. Since the topology of $X$ is linear, from $X^{\wedge}=\{1\}$ we deduce that $X$ is indiscrete whenever $X \in \mathfrak{B}$. Viceversa, indiscrete groups are minimally almost periodic, so $X \in \mathfrak{B}$ in such a case.

Finally, (d) follows from (c) since our hypotheses imply that $X$ has a linear topology ([20, Theorem II.7.7, p. 62]).

(e) According to item (a), $\Gamma_{\text {abs }}(X)=X^{\wedge}$. Hence $X \in \mathfrak{B}$ yields $X^{\wedge}=\{1\}$.

Obviously, to the equivalent conditions of item (c) of the above proposition one can add also " $X$ is minimally almost periodic".

It follows from item (b) of the next lemma that the class $\mathfrak{B}$ is stable under taking continuous homomorphic images and under taking completions.

Lemma 6.6. For topological abelian groups $X, Y$ and $\varphi \in \mathrm{CHom}(X, Y)$ one has:

(a) $\varphi^{\wedge}\left(\Gamma_{\text {abs }}(Y)\right) \subset \Gamma_{\text {abs }}(X)$.

(b) If $X \in \mathfrak{B}$ and $\varphi(X)$ is dense in $Y$, then $Y \in \mathfrak{B}$.

(c) If $\mathbf{n}(X)$ is the von-Neumann's kernel of $X$, then $X \in \mathfrak{B}$ iff $X / \mathbf{n}(X) \in \mathfrak{B}$.

Proof. Statement (a) is easy to verify.

(b) By the assumption $\Gamma_{\text {abs }}(X)=\{1\}$ and from (a) we get $\varphi^{\wedge}\left(\Gamma_{\text {abs }}(Y)\right)=\{1\}$. Now it remains to note that $\varphi^{\wedge}$ is injective by the density of $\varphi(X)$ in $Y$.

(c) The implication $X \in \mathfrak{B} \Longrightarrow X / \mathbf{n}(X) \in \mathfrak{B}$ follows from (b). The implication $X / \mathbf{n}(X) \in \mathfrak{B} \Longrightarrow X \in \mathfrak{B}$ follows from the fact that the canonical homomorphism $\varphi: X \rightarrow X / \mathbf{n}(X)$ induces an isomorphism $\varphi^{\wedge}:(X / \mathbf{n}(X))^{\wedge} \rightarrow X^{\wedge}$.

Now we prove that the class $\mathfrak{B}$ is stable also under arbitrary direct products.

Proposition 6.7. Let I be a non-empty index set and let $\left(X_{i}\right)_{i \in I}$ be a family of topological groups. Then the cartesian product $\prod_{i \in I} X_{i}$ belongs to $\mathfrak{B}$ iff $X_{i} \in \mathfrak{B}$ for every $i \in I$.

Proof. Assume that $X_{i} \in \mathfrak{B}$ for every $i \in I$. Fix $\varphi \in\left(\prod_{i \in I} X_{i}\right)^{\wedge}$. It is known (see, e.g., [4] or [15, Exercise $2.10 .4(\mathrm{~g}, \mathrm{~h})]$ ) that there is a family

$$
\left(\xi_{i}\right)_{i \in I} \in \prod_{i \in I} X_{i}^{\wedge}
$$

such that $\left|\left\{i \in I: \xi_{i} \neq 1\right\}\right|<\infty$ and

$$
\varphi(\mathbf{x})=\prod_{i \in I} \xi_{i}\left(x_{i}\right), \quad \forall \mathbf{x}=\left(x_{i}\right)_{i \in I} \in \prod_{i \in I} X_{i} .
$$

Suppose now that $\varphi \in \Gamma_{\text {abs }}\left(\prod_{i \in I} X_{i}\right)$. It is easy to see that $\xi_{i} \in \Gamma_{\text {abs }}\left(X_{i}\right), \forall i \in I$. 
From the assumption $\Gamma_{\mathrm{abs}}\left(X_{i}\right)=\{1\}, \forall i \in I$, we get that $\xi_{i}=1, \forall i \in I$. Hence $\varphi=1$ and so $\Gamma_{\text {abs }}\left(\prod_{i \in I} X_{i}\right)=\{1\}$.

The converse follows from Lemma $6.6(\mathrm{~b})$.

\section{Description of the class $\mathfrak{B}$}

Here we offer a complete description of the metrizable groups and the LCA group in $\mathfrak{B}$ (see Theorem 6.12 and Corollary 6.13). The following notion due to Enflo [17] is a cornerstone in the sequel.

Definition 6.8 ([17, p. 236]). A topological group $X$ is called locally generated if

$$
\langle V\rangle=X, \quad \forall V \in \mathcal{N}(X) .
$$

It is easy to observe that a topological group $X$ is locally generated iff

$$
X=\bigcup_{k=1}^{\infty}\left(V+\ldots k^{k \text { summands }}+V\right), \quad \forall V \in \mathcal{N}(X) .
$$

Lemma 6.9. A topological group $X$ is locally generated iff $X$ has no proper open subgroups.

Proof. If $X$ has a proper open subgroup, then obviously it is not locally generated. Conversely, if $X$ it is not locally generated, then for a symmetric open $V \in \mathcal{N}(X)$ the open subgroup $H:=\langle V\rangle$ is proper.

Corollary 6.10. If $H$ is a dense subgroup of a topological group $G$, then $H$ is locally generated iff $G$ is locally generated.

Proof. If $A$ is a proper open subgroup of $H$, then its closure is a proper open subgroup of $G$. On the other hand, if $C$ is a proper open subgroup of $G$, then $C \cap H$ is a proper open subgroup of $H$. Now Lemma 6.9 applies.

Further easy properties and known facts of the locally generated groups are collected in the next remark.

Remark 6.11. (a) By Lemma 6.9, all connected topological groups are locally generated (see also ([20, Theorem II.7.4]). On the other hand, every locally generated locally compact group is connected ([20, Corollary II.7.9]).

(b) From (a) and Corollary 6.10 one can deduce that a locally precompact group is locally generated iff its completion is connected (i.e., the locally generated locally precompact groups are precisely the dense subgroups of the connected locally compact groups). 
(c) By Corollary 6.10, the additive group of rational numbers $\mathbb{Q}$ with the usual topology is a metrizable locally generated group which is totally disconnected. A complete metrizable locally generated topological abelian group may also be totally disconnected ([17, Example 2.2.1]).

Theorem 6.12. Let $X$ be a topological abelian group.

(a) If $X \in \mathfrak{B}$, then $X$ is locally generated.

(b) If $X$ is locally generated and metrizable, then $X \in \mathfrak{B}$.

Proof. (a) Take an open subgroup $H$ of $X$. Then the quotient $X / H$ is discrete, so $X / H \in \mathfrak{B}$ by Lemma 6.6 (b). This implies that $X / H$ is a singleton by item (c) of Proposition 6.5. Hence, $H=X$ and by Lemma $6.9 X$ is locally generated.

(b) Take a character $\xi \in X^{\wedge} \backslash\{1\}$. In order to prove that $\xi \notin \Gamma_{a b s}(X)$, we must find a sequence $\left(x_{n}\right)_{n \in \mathbb{N}} \in c_{0}(X)$ such that

$$
\left(\prod_{k=1}^{n} \xi\left(x_{k}\right)\right)_{n \in \mathbb{N}}
$$

is not convergent in $\mathbb{S}$.

As $\xi \in X^{\wedge} \backslash\{1\}$, there is $x \in X$ such that $\xi(x) \neq 1$. Let $\left\{V_{1}, V_{2}, \ldots\right\}$ be a basis for $\mathcal{N}(X)$ such that $V_{n} \supset V_{n+1}, n=1,2, \ldots$. Since $X$ is locally generated,

$$
X=\bigcup_{k=1}^{\infty}\left(V_{n}+\ldots k^{k \text { summands }}+V_{n}\right), \quad \forall n \in \mathbb{N} .
$$

Thus, for a given $n \in \mathbb{N}$ we can find $k_{n} \in \mathbb{N}$ such that

$$
x \in V_{n}+\ldots k_{n} \text { summands }+V_{n} .
$$

Therefore, we can also find a finite sequence $x_{n, 1}, \ldots, x_{n, k_{n}}$ such that

$$
x=\sum_{i=1}^{k_{n}} x_{n, i} \quad \text { and } \quad x_{n, i} \in V_{n} \text { for } i=1, \ldots, k_{n} .
$$

Let

$$
m_{0}=0 \quad \text { and } \quad m_{n}:=\sum_{i=1}^{n} k_{i}, \quad n=1,2, \ldots
$$

Define now a sequence $\left(x_{j}\right)_{j \in \mathbb{N}}$ as follows: find for $j \in \mathbb{N}$ the unique $n \in \mathbb{N}$ with $m_{n-1}<j \leq m_{n}$ and put

$$
x_{j}:=x_{n, j-m_{n-1}}
$$


Clearly,

$$
\begin{aligned}
x_{1} & =x_{1,1}, \ldots, x_{m_{1}}=x_{1, m_{1}}, \\
x_{m_{1}+1} & =x_{2,1}, \ldots, x_{m_{2}}=x_{2, k_{2}}, \\
x_{m_{2}+1} & =x_{3,1}, \ldots, x_{m_{3}}=x_{3, k_{3}},
\end{aligned}
$$

and

$$
x_{j} \in V_{n}, \quad j>m_{n-1}, n=1,2,3, \ldots
$$

The last relation, since $m_{n} \rightarrow \infty$ and $\left(V_{n}\right)_{n \in \mathbb{N}}$ is a decreasing basis for $\mathcal{N}(X)$, implies that the sequence $\left(x_{j}\right)_{j \in \mathbb{N}}$ converges to zero in $X$. Now,

$$
\prod_{j=m_{n-1}+1}^{m_{n}} \xi\left(x_{j}\right)=\prod_{i=1}^{k_{n}} \xi\left(x_{n, i}\right)=\xi\left(\sum_{i=1}^{k_{n}} x_{n, i}\right)=\xi(x) \neq 1, \quad n=1,2,3, \ldots
$$

Consequently, $\left(\prod_{j=1}^{n} \xi\left(x_{j}\right)\right)_{n \in \mathbb{N}}$ is not a Cauchy sequence in $\mathbb{S}$ and hence it is not convergent.

Since connected groups are locally generated, we obtain:

Corollary 6.13. A metrizable abelian group $X \in \mathfrak{B}$ iff $X$ is locally generated. In particular, $\mathfrak{B}$ contains all connected metrizable groups.

Remark 6.14. (a) As $\mathfrak{B}$ contains all minimally almost periodic groups (see Lemma 6.6(c)), from Theorem 6.12 (a) we conclude that a minimally almost periodic group is necessarily locally generated. In $[15, \mathrm{p} .21]$ this observation is used for producing a Hausdorff group topology $\tau$ in $\mathbb{Z}^{(\mathbb{N})}$ such that $\left(\mathbb{Z}^{(\mathbb{N})}, \tau\right)$ is minimally almost periodic.

(b) According to the above corollary and to Corollary 6.10, if $X$ is a dense subgroup of a metrizable group $Y$, then $X \in \mathfrak{B}$ iff $Y \in \mathfrak{B}$. Metrizability cannot be removed here, since there exist even compact groups $Y \in \mathfrak{B}$, with a dense and sequentially closed connected subgroup $X \notin \mathfrak{B}$ (see Remark 6.16 (a)).

The following theorem implies, in particular, that the metrizability assumption can be removed from Theorem 6.12 (b) in the locally compact case (however this cannot be done in general, see Remark 6.16).

Theorem 6.15. For a locally compact abelian group X TFAE:

(i) $X \in \mathfrak{B}$.

(ii) $X$ is locally generated.

(iii) $X$ is connected. 
Proof. (i) $\Longrightarrow$ (ii) By Theorem 6.12 (a), (i) implies that $X$ is locally generated.

The implication (ii) $\Longrightarrow$ (iii) follows from [20, Corollary 7.9].

(iii) $\Longrightarrow$ (i) Take $\xi \in \Gamma_{\text {abs }}(X)$ and let us verify that $\xi=1$. To this end, consider the set

$$
A=\bigcup_{\varphi \in \mathrm{CHom}(\mathbb{R}, X)} \varphi(\mathbb{R}) .
$$

Let us see first that

$$
\left.\xi\right|_{A}=1 \text {. }
$$

Fix $\varphi \in \operatorname{CHom}(\mathbb{R}, X)$ and set $H=\varphi(\mathbb{R})$. Clearly,

$$
\left.\xi\right|_{H} \in \Gamma_{\mathrm{abs}}(H) .
$$

By Theorem 6.12(b), $\mathbb{R} \in \mathfrak{B}$. By Lemma 6.6(b),

$$
H=\varphi(\mathbb{R}) \in \mathfrak{B}
$$

too. Therefore,

$$
\left.\xi\right|_{H} \in \Gamma_{\mathrm{abs}}(H)=\{1\} \quad \text { and }\left.\quad \xi\right|_{H}=1 .
$$

Consequently (6.2) is proved.

Clearly, (6.2) implies

$$
\left.\xi\right|_{\langle A\rangle}=1 \text {. }
$$

Now, according to [20, Theorem 25.20, p. 410] the connectedness of $X$ implies that $\langle A\rangle$ is a dense subgroup of $X$. From this and (6.3) we obtain that $\xi=1$.

Remark 6.16. (a) Local compactness is essential for the implication (iii) $\Longrightarrow$ (i) of Theorem 6.15. Indeed, that implication may fail in general even for a pseudocompact group $X$. In fact, according to [19, Corollary 2.10] (see also [27, Remark 3.4]) there exists an infinite connected pseudocompact abelian group $X$ without non-trivial convergent sequences. By Proposition 6.5 (e), $X \notin \mathfrak{B}$ for such a group is MAP. Consistent examples of connected countably compact groups without infinite compact subsets can be found in [16, Corollary 2.21] (note that in both cases the groups are sequentially complete). In connection with item (b) of Remark 6.14, we note that the completion $Y$ of $X$ is a compact connected group, so $Y \in \mathfrak{B}$ according to Theorem 6.15.

(b) From the proof of Theorem 6.12 (b) and (ii) $\Longrightarrow$ (i) of Theorem 6.15 it follows that if a locally generated Hausdorff topological abelian group $X$ is either complete metrizable or locally compact, then $c_{0}(X) \neq \operatorname{ss}(X)$.

Another class of non-metrizable groups in $\mathfrak{B}$ can be obtained from Proposition 6.7 . 


\section{Applications of the class $\mathfrak{B}$}

\section{Groups with countable dual}

Proposition 7.1. Let $X \neq\{0\}$ be a compact abelian group and $G:=\left(c_{0}(X), \mathfrak{u}_{0}\right)$.

(a) If $X$ is connected, then $\left|G^{\wedge}\right|=\left|X^{\wedge}\right|$.

(b) If $X$ is connected and metrizable, then $\left|G^{\wedge}\right|=\aleph_{0}$.

(c) If $X$ a metrizable and disconnected, then $\left|G^{\wedge}\right|=c$.

Proof. Statement (a) follows from Corollary 6.4 via implication (iii) $\Longrightarrow$ (i) of Theorem 6.15.

Statement (b) follows from Theorem $6.12(\mathrm{~b})$ and the equality $\left|X^{\wedge}\right|=\aleph_{0}$ (Proposition 2.3).

(c) It is easy to verify that

$$
\xi \in \Gamma_{\mathrm{abs}}(X) \Longrightarrow\{1, \xi\}^{\mathbb{N}} \subset c_{0}(X)^{\beta} .
$$

Since $X$ is compact metrizable, by Proposition 5.1 (c) we get

$$
\xi \in \Gamma_{\text {abs }}(X), \mathbf{h} \in\{1, \xi\}^{\mathbb{N}} \Longrightarrow \chi_{\mathbf{h}} \in G^{\wedge} .
$$

Now by Theorem $6.15, X \notin \mathfrak{B}$ and consequently there exists $\xi \in \Gamma_{\mathrm{abs}}(X)$, with $\xi \neq 1$. Then $\left|\{1, \xi\}^{\mathbb{N}}\right|=c$. Therefore

$$
\left\{\chi_{\mathbf{h}}: \mathbf{h} \in\{1, \xi\}^{\mathbb{N}}\right\} \subset G^{\wedge}
$$

and since the correspondence $\mathbf{h} \mapsto \chi_{\mathbf{h}}$ is injective, we get that $\left|G^{\wedge}\right| \geq \mathbf{c}$.

The following statement shows that Proposition 7.1 (b) is the best possible in the class of locally compact groups.

Proposition 7.2. For an infinite locally compact Hausdorff topological abelian group X TFAE:

(i) $X$ is compact connected and metrizable.

(ii) $\left|\left(c_{0}(X), \mathfrak{u}_{0}\right)^{\wedge}\right|=\aleph_{0}$.

Proof. The implication (i) $\Longrightarrow$ (ii) is a consequence of Proposition 7.1 (b).

(ii) $\Longrightarrow$ (i) Let us see first that $X$ is compact and metrizable. To this end, write $G:=\left(c_{0}(X), \mathfrak{u}_{0}\right)$. Let $\varphi: G \rightarrow X$ be the first projection, i.e., the mapping which sends $\left(x_{n}\right)_{n \in \mathbb{N}}$ to $x_{1}$. Then $\varphi^{\wedge}: X^{\wedge} \rightarrow G^{\wedge}$ is injective. So $\left|G^{\wedge}\right|=\aleph_{0}$ implies that $\left|X^{\wedge}\right| \leq \aleph_{0}$ and by Proposition 2.3 we get that the group $X$ is compact metrizable. The fact that $X$ is connected derives from Proposition 7.1 (c) and the equality $\left|\left(c_{0}(X), \mathfrak{u}_{0}\right)^{\wedge}\right|=\aleph_{0}$. 
Now we are in a position to prove our Main Theorem. Let us note that it provides a characterization of the connected groups in the class of all compact metrizable abelian groups.

Proof of the Main Theorem. We have to prove that for an infinite compact metrizable abelian group $X$ TFAE:

(i) $X$ is connected.

(ii) $\left(c_{0}(X), \mathfrak{u}_{0}\right)^{\wedge}=\left(X^{\wedge}\right)^{(\mathbb{N})}$.

(iii) $\left|\left(c_{0}(X), \mathfrak{u}_{0}\right)^{\wedge}\right|=\aleph_{0}$.

(iv) $\left|\left(c_{0}(X), \mathfrak{u}_{0}\right)^{\wedge}\right|<\mathfrak{c}$.

The implication (iii) $\Longrightarrow$ (iv) is trivial, the implications (i) $\Longrightarrow$ (ii) $\Longrightarrow$ (iii) follow from Proposition 7.1 (a) and the implication (iv) $\Longrightarrow$ (i) follows from Proposition 7.1 (c).

In order to prove the last assertion, assume that $X$ is connected. Then, by Proposition $3.4,\left(c_{0}(X), \mathfrak{u}_{0}\right)$ is connected.

The following theorem provides a wide class of precompact metrizable groups which are not Mackey in LQC, extending thus the result of Proposition 5.2 and Theorem 1.8.

Theorem 7.3. Let $X$ be a non-trivial compact connected metrizable group. Then we have:

(a) $\left(c_{0}(X), \mathfrak{p}_{0}\right)^{\wedge}=\left(c_{0}(X), \mathfrak{u}_{0}\right)^{\wedge}$.

(b) $\mathfrak{u}_{0}$ is a locally quasi-convex connected Polish group topology compatible for $\left(c_{0}(X), \mathfrak{p}_{0}\right)$.

(c) $\left(c_{0}(X), \mathfrak{p}_{0}\right)$ is a precompact metrizable connected non-Mackey group.

Proof. (a) Since $\mathfrak{p}_{0} \leq \mathfrak{u}_{0}$, we have

$$
\left(c_{0}(X), \mathfrak{p}_{0}\right)^{\wedge} \subseteq\left(c_{0}(X), \mathfrak{u}_{0}\right)^{\wedge} .
$$

To prove the converse inclusions, fix an arbitrary $\kappa \in\left(c_{0}(X), \mathfrak{u}_{0}\right)^{\wedge}$. By the Main Theorem, there exists $\mathbf{h}=\left(\xi_{n}\right)_{n \in \mathbb{N}} \in\left(X^{\wedge}\right)^{(\mathbb{N})}$ such that $\kappa=\chi_{\mathbf{h}}$. Consequently,

$$
\kappa \in\left(c_{0}(X), \mathfrak{p}_{0}\right)^{\wedge}
$$

and (a) is proved.

(b) By Proposition 3.4, $\mathfrak{u}_{0}$ is a locally quasi-convex Polish group topology, and by (a) it is compatible for $\left(c_{0}(X), \mathfrak{p}_{0}\right)$. Connectedness follows from Proposition $3.4(\mathrm{c})$. 
(c) $\left(c_{0}(X), p_{0}\right)$ is a precompact metrizable group because it is a topological subgroup of the compact metrizable group $\left(X^{\mathbb{N}}, \mathfrak{p}\right)$. It is not a Mackey group in LQC because by (b) $\mathfrak{u}_{0}$ is a locally quasi-convex group topology, compatible for $\left(c_{0}(X), \mathfrak{p}_{0}\right)$ and strictly finer than $\mathfrak{p}_{0}$ (Proposition 3.1 $\left.\left(\mathrm{a}_{1}\right)\right)$. Connectedness follows from item (b), as $p_{0} \leq \mathfrak{u}_{0}$.

Remark 7.4. (a) Using the ideas from the above proofs it can be established that for every non-trivial compact connected metrizable group $X$ the group $c_{0}(X)$ carries exactly $c$ many connected separable metrizable locally quasi-convex non-Mackey topologies compatible with $\mathfrak{p}_{0}$. A detailed proof will be given elsewhere.

(b) One cannot use the group $G=\left(X^{\mathbb{N}}, \mathfrak{u}\right)$ instead of $\left(c_{0}(X), \mathfrak{u}_{0}\right)$ in Theorem 7.3 since

$$
\left|G^{\wedge}\right|=2^{\mathrm{c}}
$$

This follows from the following more general fact that we mention without proof: if $X \neq\{0\}$ is a compact group and $G=\left(X^{\mathbb{N}}, \mathfrak{u}\right)$, then

$$
|\mathrm{CHom}(G, X)| \geq 2^{\mathrm{c}} \text {. }
$$

\section{Open questions}

The problem of existence of $\mathcal{E}$-Mackey topologies is not discussed in this paper. It was raised in [10, Question 1, p. 275] for $\mathscr{G}=\mathrm{LQC}$, and it still remains open even for that case.

It follows from [10, Proposition 5.4] that every non-meager $(G, \mu) \in \operatorname{LCS}$ is a Mackey group in LQC.

Conjecture 8.1. Every metrizable $(G, \mu) \in$ LCS is a Mackey group in LQC.

We do not even know if $\mathbb{R}^{(\mathbb{N})}$ with the topology induced from the product space $\mathbb{R}^{\mathbb{N}}$ is a Mackey group in LQC.

Conjecture 8.2. Proposition 5.1 (c) remains true for a (not necessarily complete metrizable) topological abelian group.

It is clear that if $G$ is a discrete group, then $G$ is a Mackey group in MAP.

Conjecture 8.3. If $G \in$ MAP is a Mackey group in MAP, then $G$ is a discrete group.

Conjecture 8.3 in terms of the Mackey topology can be reformulated as follows. 
Conjecture 8.4. If for a given precompact topological group $(G, v)$ there exists the MAP-Mackey topology in $G$ associated with $v$, then $v$ is the finest precompact group topology in $G$.

Finally, we reformulate Conjecture 8.3 for LCA groups:

Conjecture 8.5. If $G \in \mathrm{LCA}$ is a Mackey group in MAP, then $G$ is a discrete group. In particular, $\mathbb{R}$ is not a Mackey group in MAP.

Remark 8.6. According to Example 1.7, a non-complete precompact metrizable $(G, \mu)$ can be a Mackey group in LQC. It is also known that every locally pseudocompact $(G, \mu)$ is a Mackey group in LQC (cf. [13]). An internal description of groups $(G, \tau) \in \mathrm{LQC}$ which are Mackey in LQC is unknown.

Acknowledgments. We thank the referee for the careful reading of our manuscript, pointing out some errors and giving good hints of how to improve the text.

\section{Bibliography}

[1] H. Anzai and S. Kakutani, Bohr compactifications of a locally compact Abelian group. II, Proc. Imp. Acad. Tokyo 19 (1943), 533-539.

[2] L. Aussenhofer, Contributions to the duality theory of abelian topological groups and to the theory of nuclear groups, Diss. Math. CCCLXXXIV, Warsaw, 1999.

[3] L. Aussenhofer and D. de la Barrera, Linear topologies on $\mathbb{Z}$ are not Mackey topologies, preprint (2011), http://arxiv .org/abs/1107.2661.

[4] W. Banaszczyk, Additive Subgroups of Topological Vector Spaces, Lecture Notes in Mathematics 1466, Springer-Verlag, Berlin, 1991.

[5] M. Barr and H. Kleisli, On Mackey topologies in topological abelian groups, Theory Appl. Categ. 8 (2001), 54-62

[6] G. Bennett and N. J. Kalton, Inclusion theorems for K-spaces, Canad. J. Math. 25 (1973), 511-524.

[7] F. G. Bonales, F. J. Trigos-Arrieta and R. Vera Mendoza, A Mackey-Arens theorem for topological abelian groups, Bol. Soc. Mat. Mexicana (3) 9 (2003), no. 1, 79-88.

[8] N. Bourbaki, General Topology, Volume I, Hermann, Paris VI, 1966.

[9] N. Bourbaki, General Topology, Volume II, Hermann, Paris VI, 1966.

[10] M. J. Chasco, E. Martín-Peinador and V. Tarieladze, On Mackey topology for groups, Stud. Math. 132 (1999), no. 3, 257-284. 
[11] W. W. Comfort and K. A. Ross, Topologies induced by groups of characters, Fund. Math. 55 (1964), 283-291.

[12] W. W. Comfort and K. A. Ross, Pseudocompactness and uniform continuity in topological groups, Pacific J. Math. 16 (1966), 483-496.

[13] L. De Leo, D. Dikranjan, E. Martín-Peinador and V. Tarieladze, Duality theory for groups revisited: g-barrelled, Mackey and Arens groups, preprint (2009).

[14] D. Dikranjan, E. Martín-Peinador and V. Tarieladze, A class of metrizable locally quasi-convex groups which are not Mackey, preprint (2010), http://arxiv.org/ abs/1012.5713.

[15] D. Dikranjan, I. Prodanov and L. Stoyanov, Topological Groups: Characters, Dualities and Minimal Group Topologies, Pure and Applied Mathematics 130, Marcel Dekker, New York, 1989.

[16] D. Dikranjan and D. Shakhmatov, Forcing hereditarily separable compact-like group topologies on abelian groups, Topology Appl. 151 (2005), no. 1-3, 2-54.

[17] P. Enflo, Uniform structures and square roots in topological groups, Israel J. Math. 8 (1970), 230-252.

[18] S. S. Gabriyelyan, Groups of quasi-invariance and the Pontryagin duality, Topology Appl. 157 (2010), no. 18, 2786-2802.

[19] J. Galindo and S. Garcia-Ferreira, Compact groups containing dense pseudocompact subgroups without non-trivial convergent sequences, Topology Appl. 154 (2007), no. 2, 476-490.

[20] E. Hewitt and K. A. Ross, Abstract Harmonic Analysis I, Die Grundlehren der Mathematischen Wissenschaften 115, Springer-Verlag, Berlin, 1963.

[21] S. Kaplan, Extension from the Pontryagin duality I: Infinite products, Duke Math. J. 15 (1948), 649-658.

[22] J. L. Kelley and I. Namioka, Linear Topological Spaces, Springer-Verlag, New York, 1963.

[23] J.W. Nienhuys, A solenoidal and monothetic minimally almost periodic group, Fund. Math. 73 (1971/72), no. 2, 167-169.

[24] S. Rolewicz, Some remarks on monothetic groups, Colloq. Math. XIII (1964), 2829.

[25] H. H. Schaefer, Topological Vector Spaces, The Macmillan Co., New York, CollierMacmillan Ltd., London, 1966.

[26] L. J. Sulley, On countable inductive limits of locally compact abelian groups, J. Lond. Math. Soc. (2) 5 (1972), 629-637.

[27] M. Tkachenko, Self-duality in the class of precompact groups, Topology Appl. 156 (2009), no. 12, 2158-2165. 
[28] N. T. Varopoulos, Studies in harmonic analysis, Proc. Cambridge Philos. Soc. 60 (1964), 465-516.

[29] N. Y. Vilenkin, The theory of characters of topological Abelian groups with boundedness given (in Russian), Izvestiya Akad. Nauk SSSR. Ser. Mat. 15 (1951), 439-162.

[30] A. Weil, L'intégration dans les groupes topologiques et ses applications, Actualités scientifiques et industrielles 869, Hermann \& Cie, Paris, 1940.

Received September 20, 2011; revised December 27, 2011.

\section{Author information}

Dikran Dikranjan, Department of Mathematics and Computer Science,

University of Udine, Via delle Scienze, 208-Loc. Rizzi, 33100 Udine, Italy.

E-mail: dikranja@dimi .uniud.it

Elena Martín-Peinador, Departamento de Geometría y Topología,

Universidad Complutense de Madrid, 28040 Madrid, Spain.

E-mail: em_peinador@mat.ucm.es

Vaja Tarieladze, Niko Muskhelishvili Institute of Computational Mathematics, 0160 Tbilisi, Georgia.

E-mail: vajatarieladze@yahoo.com 